\title{
Preventing neuronal edema increases network excitability after traumatic brain injury
}

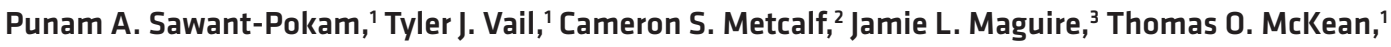 \\ Nick O. McKean, ${ }^{1}$ and K.C. Brennan ${ }^{1}$ \\ 'Department of Neurology, School of Medicine, and '2Anticonvulsant Drug Development Program, Department of Pharmacology and Toxicology, College of Pharmacy, University of Utah, Salt Lake City, \\ Utah, USA. ${ }^{3}$ Neuroscience Department, Tufts University School of Medicine, Boston, Massachusetts, USA.
}

\begin{abstract}
Edema is an important target for clinical intervention after traumatic brain injury (TBI). We used in vivo cellular resolution imaging and electrophysiological recording to examine the ionic mechanisms underlying neuronal edema and their effects on neuronal and network excitability after controlled cortical impact (CCI) in mice. Unexpectedly, we found that neuronal edema 48 hours after $\mathrm{CCl}$ was associated with reduced cellular and network excitability, concurrent with an increase in the expression ratio of the cation-chloride cotransporters (CCCS) NKCC1 and KCC2. Treatment with the CCC blocker bumetanide prevented neuronal swelling via a reversal in the NKCC1/KCC2 expression ratio, identifying altered chloride flux as the mechanism of neuronal edema. Importantly, bumetanide treatment was associated with increased neuronal and network excitability after injury, including increased susceptibility to spreading depolarizations (SDs) and seizures, known agents of clinical worsening after TBI. Treatment with mannitol, a first-line edema treatment in clinical practice, was also associated with increased susceptibility to SDs and seizures after $\mathrm{CCl}$, showing that neuronal volume reduction, regardless of mechanism, was associated with an excitability increase. Finally, we observed an increase in excitability when neuronal edema normalized by 1 week after $\mathrm{CCl}$. We conclude that neuronal swelling may exert protective effects against damaging excitability in the aftermath of TBI and that treatment of edema has the potential to reverse these effects.
\end{abstract}

\section{Introduction}

Traumatic brain injury (TBI) is a major cause of death and morbidity in both military and civilian populations (1). Because of the high incidence and the disability associated with TBI, there is an urgent need for a better understanding of the underlying mechanisms. TBI unleashes complex, temporally overlapping pathophysiological events contributing to both primary (directly associated with trauma) and secondary injuries (2). Edema is one of the most prominent secondary injuries - it is observed within minutes, can persist for many days, and is the principal cause of death after TBI (3). Edema is thus an important target for intervention, though the primary treatments have not changed in decades and often do not meet with success (4).

Mechanisms of edema have been extensively investigated after TBI (3); however, there has been comparatively little examination of the neuronal $(5,6)$, rather than astrocytic, vascular or bulk tissue response. This is surprising, because effects on neuronal function ultimately determine network activity after injury. Edema in uninjured brain slices is known to acutely enhance seizure-like activity (7). However, the data after TBI appear contradictory: recordings in brain slices show enhanced neuronal membrane (8) and network excitability (9), whereas in vivo multicellular recordings show reduced local network activity (10). This

Conflict of interest: The authors have declared that no conflict of interest exists. Copyright: () 2020, American Society for Clinical Investigation.

Submitted: November 5, 2019; Accepted: August 6, 2020; Published: October 12, 2020. Reference information: J Clin Invest. 2020;130(11):6005-6020.

https://doi.org/10.1172/JCl134793. apparent contradiction underlines the need to study the effects of TBI on neuronal function at the single-cell level in vivo.

Seizures have long been associated with worsening of neurological status after brain injury $(11,12)$. More recently, spreading depolarizations (SDs) have become widely recognized as mechanisms of diseases as diverse as TBI, stroke, subarachnoid hemorrhage, and migraine (13). Electrophysiological recordings in brain-injured patients demonstrate slow potential changes accompanied by a depression in electrocorticographic activity $(14,15)$ consistent with SDs, which are also often associated with seizure-like activity $(16,17)$. In experimental models, SDs are observed immediately after injury, with electrophysiological depression lasting almost 3 hours after injury (18). SDs and seizures are also seen together in experimental preparations (19-24). When either SDs or seizures occur in the injured brain, they impose a substantial metabolic burden that exacerbates tissue damage $(13,25,26)$. Indeed, SDs and seizures in patients with TBI are associated with worsened clinical outcomes $(11,12,27)$. Though SD and seizure incidence coincide with a period of tissue edema $(18,28)$, the relationship between these two entities and edema is essentially unknown.

We used cellular resolution techniques - in vivo whole-cell electrophysiological recordings and 2-photon imaging - to test the effects of TBI-associated edema on neuronal and network function. We focused on the time point 48 hours after TBI in mice, a time of maximal edema in rodents $(29,30)$ and humans $(31)$. To our initial surprise, we recorded robust reductions in neuronal and network excitability that coincided with neuronal swelling and increases in the $\mathrm{Na}^{+}-\mathrm{K}^{+}-\mathrm{Cl}^{-}$cotransporter $1 / \mathrm{K}^{+}-\mathrm{Cl}^{-}$cotransporter 
2 (NKCC1/KCC2) ratio. Reducing neuronal swelling with the cation-chloride cotransporter (CCC) inhibitor bumetanide resulted in increased membrane and synaptic excitability, increased local and sensory network activity, and increased susceptibility to SDs and seizures. Moreover, decreasing neuronal swelling with mannitol, a first-line clinical treatment, led to increased SD and seizure susceptibility. Similarly, when neuronal swelling resolved 1 week after controlled cortical impact (CCI) TBI, we found that network excitability was increased. We concluded that, instead of increasing excitation, neuronal edema may actually constrain it, at least between 2 hours and 48 hours after injury. These data generate a more nuanced view of the edema process, which has been presumed to be universally harmful, and may argue for more targeted approaches to the treatment of edema.

\section{Results}

Neuronal edema and CCC expression changes after CCI. We performed 2-photon imaging of neuronal volume and concurrent Western blot experiments to examine CCC expression 2 hours, 48 hours, and 1 week after CCI TBI, in the hind paw region of the somatosensory cortex of urethane-isoflurane-anesthetized mice. We chose the CCI TBI model because it is extensively validated, and it reproduces the changes, including edema, reported in military and civilian head injuries (2). We used Thy1-GCaMP6s mice (32), which express the calcium indicator GCaMP6s in excitatory projection neurons, to measure neuronal volume.

We observed a characteristic pattern of injury following CCI, with an area of cortical cavitation encircled by dead or dying cells adjacent to a surrounding region of structurally intact tissue (ref. 33 and Figure 1, A and B). We focused on this surrounding region, because we wished to examine surviving neurons and because this region is thought to be a site of hyperexcitability at chronic time points after injury (34). Sham-treated animals showed neither cortical lesions nor cell death, despite also having undergone a craniotomy.

We used 2-photon imaging for optical sectioning of layer 2/3 sensory cortex in Thy1-GCaMP6s mice (Figure 1C) (32). We measured the cross-sectional area of neuronal somata from maximum-intensity projection images as a proxy for neuronal volume (see Methods and Supplemental Results and Discussion, Cell size measurements during calcium activity; supplemental material available online with this article; https://doi.org/10.1172/ JCI134793DS1). The somatic area was significantly larger in neurons of CCI- versus sham-treated mice 48 hours after CCI, but not at 1 week (48 hours: $P=0.006,2$-sided, unpaired $t$ test; $n=$ 68-75 neurons, $n=4-5$ mice per group; 1 week: $P>0.05$, 2-sided, unpaired $t$ test; $n=37-67$ neurons, $n=4$ mice per group) (Figure $1, \mathrm{D}$ and $\mathrm{E})$. These data provide what we believe is the first in vivo evidence of cellular resolution of neuronal (rather than bulk tissue) edema after TBI.

Neurons do not contain aquaporin 4 water channels (35), which mediate cellular swelling in astrocytes and are a major mechanism of bulk tissue edema formation $(3,36)$. Although neuronal edema is less understood, neurons can generate volume increases, primarily through increased expression or function of the sodium potassium chloride cotransporter NKCC1 (mediating $\mathrm{Na}^{+}, \mathrm{K}^{+}, 2 \mathrm{Cl}^{-}$, and $\mathrm{H}_{2} \mathrm{O}$ entry) (37). Volume decreases occur via the potassium chloride cotransporter $\mathrm{KCC} 2$ (mediating $\mathrm{K}^{+}, \mathrm{Cl}^{-}$, and $\mathrm{H}_{2} \mathrm{O}$ efflux) (37). Increases in NKCC1 and decreases in KCC2 expression have been reported after TBI $(38,39)$, either of which could be associated with the edema phenotype. Thus, we performed Western blot analysis of NKCC1 and KCC2 expression in the hind paw region of the sensory cortex.

We observed substantial changes in CCC protein expression in animals subjected to CCI compared with sham-treated animals. We found that KCC2 protein expression was reduced in CCI-treated mice 2 hours, 48 hours, and 1 week after TBI compared with sham-treated mice, and the changes were greatest at 2 hours, intermediate at 48 hours, and smaller but still statistically significant 1 week after injury (Figure 1, F-H). Meanwhile NKCC1 levels were unchanged throughout (KCC2 at 2 hours: $P=0.004$, 2-sided Mann-Whitney $U$ test; NKCC1, $P>0.05$, 2-sided Mann-Whitney $U$ test; $n=5-6$ mice per group; KCC2 at 48 hours: $P=0.004$, 2-sided Mann-Whitney $U$ test; NKCC1, $P>0.05$, 2-sided Mann-Whitney $U$ test; $n=6$ mice per group; KCC2 at 1 week: $P=0.04,2$-sided Mann-Whitney $U$ test; NKCC1, $P>0.05$, 2-sided Mann-Whitney $U$ test; $n=6$ mice per group) (Figure $1, \mathrm{~F}-\mathrm{H}$ ). A reduction in KCC2 expression caused an increase in the NKCC1/KCC2 ratio, which would be expected to result in net chloride and water influx (40), leading to neuronal edema.

Neuronal swelling coincides with reduced intrinsic membrane excitability in CCI neurons. Next, we performed somatic current-clamp recordings from layer $2 / 3$ pyramidal neurons in shamand CCI-treated mice 48 hours after injury, with the rationale that this is the time point of maximum edema in both rodents $(29,30)$ and humans (31) and, in humans, the time point at which the clinical consequences of edema are most apparent (Figure 2A). The resting membrane potential (RMP) did not differ between CCIand sham-treated groups $(P>0.05,2$-sided, unpaired $t$ test; $n=$ 9-10 neurons, $n=6-10$ mice per group) (Figure $2 \mathrm{~B}$ ). However, neurons of CCI-treated mice had significantly larger membrane capacitance $\left(C_{m}\right)$ than did those of sham-treated mice ( $P=0.01,2$-sided, unpaired $t$ test; $n=14-15$ neurons, $n=10-12$ mice per group) (Figure 2C). A larger $\mathrm{C}_{\mathrm{m}}$ was consistent with the larger neuronal area (Figure 1D, Supplemental Figure 2, Supplemental Figure 4A, Supplemental Figure 5A, and refs. 41-43) observed in CCI-treated mice, and this would also be expected to coincide with reductions in input resistance $\left(R_{i n}\right)$, which in turn would likely be associated with reductions in intrinsic membrane excitability (41-43). We found that neurons from CCI-treated mice indeed had smaller $R_{\text {in }}$ than did neurons from sham-treated mice ( $P=0.007,2$-sided Mann-Whitney $U$ test) (Figure 2D). We confirmed that differences in $R_{\text {in }}$ were not due to changes in access resistance (CCI: 20.66 $\pm 0.39 \mathrm{M} \Omega$, sham: $20.84 \pm 0.36 \mathrm{M} \Omega ; P>0.05$, 2-sided, unpaired $t$ test), which could have been a confounding explanation.

As expected with reduced $R_{i n}$, neurons from CCI-treated mice showed a decrease in the number of action potentials (APs) elicited by depolarizing current steps compared with those from sham-treated mice (Figure 2, E and F), resulting in a significantly decreased slope of the input/output (I-V) curve in neurons from CCI-treated mice relative to those from sham-treated mice $(P=$ 0.001, 2-sided Mann-Whitney $U$ test) (Figure 2G). CCI neurons also had an increased rheobase relative to sham neurons $(P=$ 0.04, 2-sided, unpaired $t$ test) (Figure $2 \mathrm{H}$ ). Our analysis of intrin- 
A

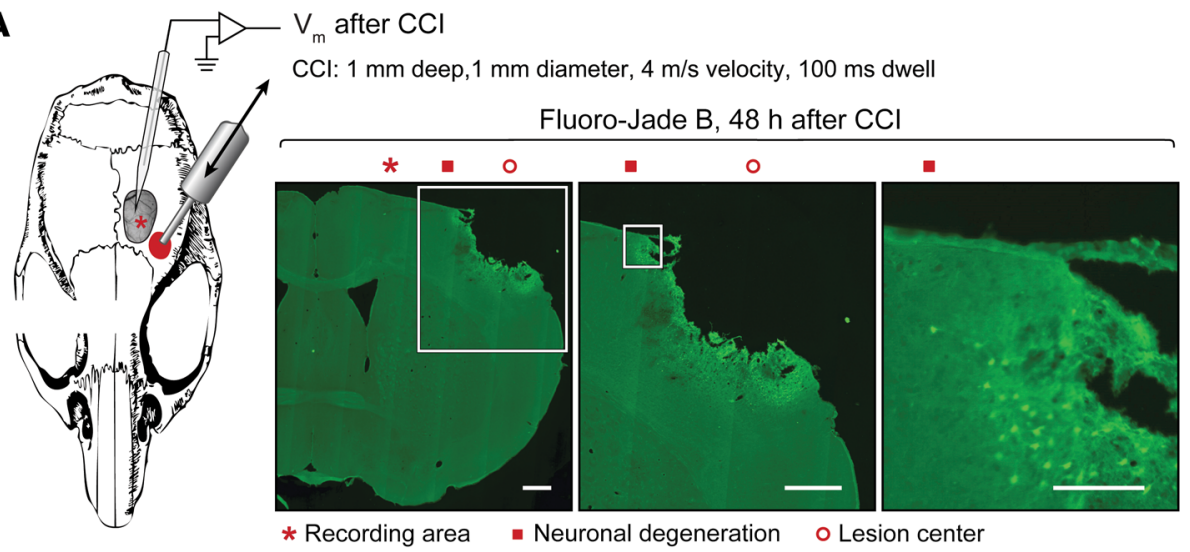

B

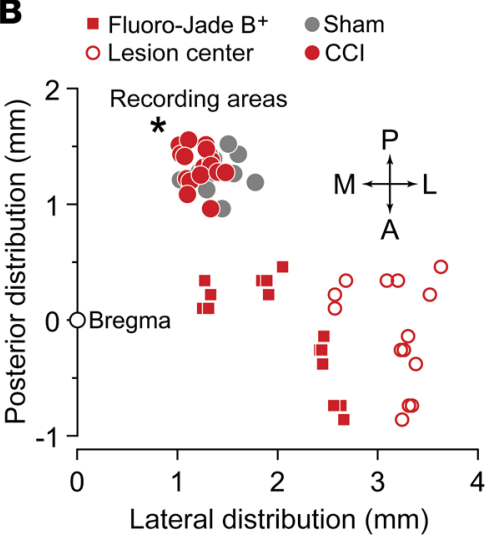

C

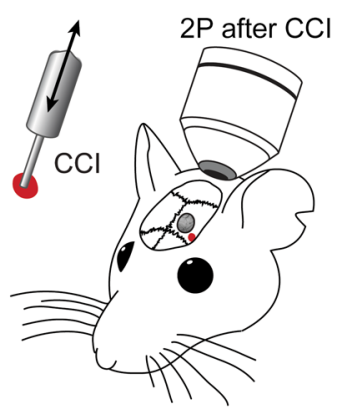

$\mathbf{F}$
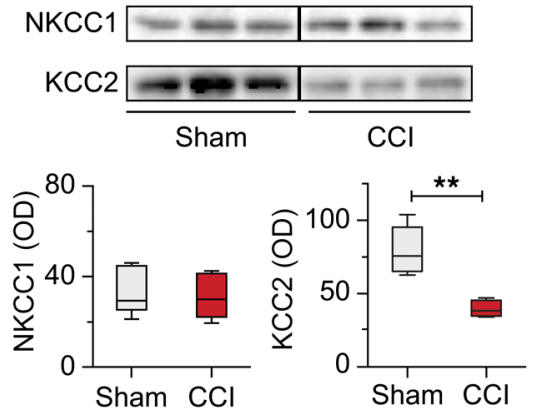

D

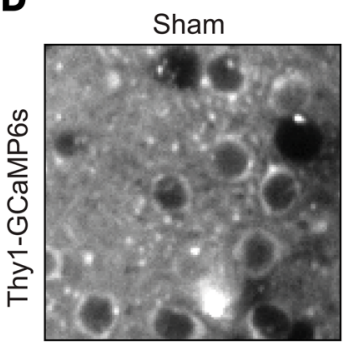

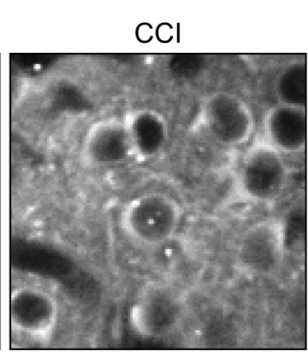

$48 \mathrm{~h}$ after $\mathrm{CCl}$
G NKCC1 $\mathrm{KCC} 2$

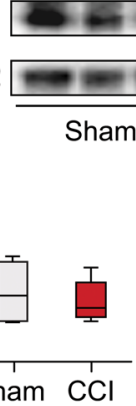

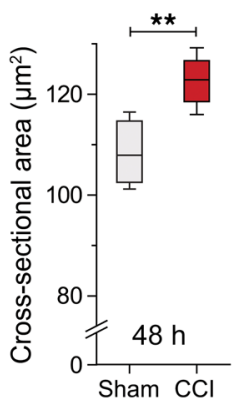

E

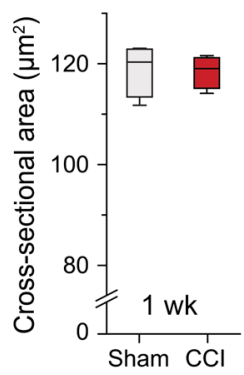

Figure 1. Neuronal volume measurements and CCC expression in the perilesional area $\mathbf{2}$ hours, $\mathbf{4 8}$ hours, and 1 week after injury. (A) Schematic showing a cranial window preparation with the CCI location (red) as well as the location for whole-cell and 2-photon recordings (red asterisk). Images show a cortical cavity at the injury site (red circles), but no overt injury to underlying structures was observed. Scale bars: $500 \mu \mathrm{m}$ and $100 \mu \mathrm{m}$ (enlarged inset shown in the third image). Tissue was stained with FJB, which labeled degenerating neurons after injury (red squares; $n=5$ mice per group). (B) Plot quantifying recording sites in relation to the injury sites and FJB-positive staining. Neuronal degeneration on FJB staining extended approximately $1.5 \mathrm{~mm}$ from the lesion center; whole-cell and 2-photon recordings were located just outside this region in a "penumbral" area with an intact cortical structure and no overt neuronal injury ( $n=4-5$ mice per group). (C) Schematic of the experimental design for 2-photon excitation imaging (2P) after CCI. (D) 2-Photon microscopic images of representative neurons in sham- and CCI-treated Thy1-GCaMP6s mice showing morphological features of pyramidal neurons in layer $2 / 3$ cortex. Scale bar: $20 \mu \mathrm{m}$. Plot shows that CCI-treated mice had significant increases in neuronal cross-sectional area 48 hours after CCI ${ }^{* *} P=0.006$, 2-sided, unpaired $t$ test; $n=68-75$ neurons, $n=4-5$ mice per group). (E) Plot shows no changes in neuronal cross-sectional area between sham-treated and CCI-treated Thy1-GCaMP6s mice at 1 week ( $P>0.05,2$-sided, unpaired $t$ test; $n=37-67$ neurons, $n=4$ mice per group). (F-H) Western blotting for NKCC1 and KCC2 two hours, 48 hours, and 1 week after CCI or sham treatment, respectively. Optical density (OD) analysis showed no change in NKCC1 but significant decreases in KCC2 expression at those time points. Plots showing OD (F) 2 hours after CCI or sham treatment $\left(\mathrm{NKCC1}, P>0.05, \mathrm{KCC} 2,{ }^{* *} P=0.004\right.$; 2-sided Mann-Whitney $U$ test; $n=5$-6 mice per group); (C) 48 hours after $\mathrm{CCI}$ or sham treatment (NKCC1, $P>0.05, \mathrm{KCC2},{ }^{* *} P=0.004 ; 2$-sided MannWhitney $U$ test; $n=6$ mice per group); and $(\mathbf{H}) 1$ week after $C C I$ or sham treatment (NKCC1, $P>0.05, \mathrm{KCC} 2,{ }^{*} P=0.04 ; 2$-sided Mann-Whitney $U$ test; $n=6$ mice per group). All lanes within the blots were run on the same gels.

sic membrane properties of neurons thus revealed substantial decreases in excitability after injury, concurrent with an increase in neuronal cross-sectional area.

Spontaneous excitatory transmission is decreased after CCI. We examined excitatory synaptic activity after $\mathrm{CCI}$ with in vivo voltage-clamp recordings in layer $2 / 3$ neurons from CCI- and sham-treated animals (Figure 2I). Inhibitory currents were not reliably obtainable, possibly because of the depolarized holding potential required for these recordings. The excitatory postsynaptic current (EPSC) inter-event interval (IEI) was significantly lon- 
ger in CCI than in sham neurons $(P=0.03,2$-sided Mann-Whitney $U$ test; $n=6-7$ neurons, $n=6-7$ mice per group) (Figure 2, J and $\mathrm{K})$. We detected no difference in the area under the curve (AUC) of EPSCs between neurons from CCI-treated or sham-treated animals ( $P>0.05$, 2-sided Mann-Whitney $U$ test) (Figure $2 \mathrm{~K})$. The reduced frequency of EPSCs is consistent with a reduction in the efficacy of synaptic inputs due to decreased input resistance after TBI (see Supplemental Results and Discussion, Mechanisms of reduced excitatory input onto pyramidal neurons after TBI).

Up-state AUC and AP frequency are reduced 48 hours after CCI. We next examined local network activity by measuring up-state responses in urethane-isoflurane-anesthetized animals (Figure 3A). Up states are generated through barrages of recurrent synaptic excitation, with strong synaptic connections causing synchronization of connected neurons $(44,45)$. Up states occur spontaneously in anesthetized animals and show periods of enhanced synaptic activity and membrane depolarization associated with enhanced AP firing, alternating with down states with more quiescent activity $(46,47)$.

Layer 2/3 pyramidal neurons from CCI- and sham-treated mice exhibited typical up- and down-state fluctuations in membrane potential. Though we observed no change in the frequency of up states (IEI; $P>0.05$, 2-sided, unpaired $t$ test; $n=12$ neurons, $n=8-10$ mice per group) (Figure $3 \mathrm{~B}$ ), the AUC of up-state events was smaller in the CCI-treated group relative to the sham-treated group ( $P=0.007,2$-sided, unpaired $t$ test) (Figure $3 \mathrm{C}$ ).

We observed spontaneous APs during up states, in a range $(0-0.2 \mathrm{~Hz})$ that was consistent with the AP frequency recorded under similar conditions in vivo $(46,48)$. Neurons from CCI-treated animals had a lower AP frequency than did sham-treated neurons $(P=0.01,2$-sided Mann-Whitney $U$ test; $n=4-5$ neurons, $n=$ 4-5 mice per group) (Figure 3, A and D), consistent with a decrease in net neuronal excitability and excitatory output after TBI.

The up-state area can be affected by both intrinsic (49) and synaptic $(45,50,51)$ mechanisms (ref. 52 and see Supplemental Results and Discussion, Possible role for potassium and Ih currents in excitability changes after TBI). The AP rate can be affected by similar factors, as well as by losses of excitatory neurons (synapses) after injury and changes in inhibition $(51,53)$. Given the large change in intrinsic membrane excitability, we hypothesized that intrinsic mechanisms contributed more substantially to the up-state and AP phenotypes, but we tested this more explicitly (see below).

Sensory-evoked activity is decreased 48 hours after CCI. In vivo animal models of TBI show reductions in sensory network activity as measured by reduced whisker-evoked neuronal c-fos activation and extracellularly recorded AP firing (10). In contrast, ex vivo studies show increased membrane (8) and local network excitability (9). We used in vivo whole-cell recordings to allow examination of both cell- and circuit-based physiology (Figure $3 \mathrm{E}$ ), as well as to address the apparent contradiction between in vivo and ex vivo data. We measured the response to electrical stimulation ( $5 \mathrm{~Hz}, 10$ stimuli, $1 \mathrm{~ms}$ duration) of the contralateral hind paw in layer $2 / 3$ of the hind paw cortex. We recorded significantly smaller amplitude excitatory postsynaptic potentials (EPSPs) in CCI neurons than in sham neurons for the whole duration of the train $(P<0.0001,2-$ way ANOVA; $n=6-9$ neurons, $n=$
6-8 mice per group) (Figure 3F). In addition, analysis of sensoryevoked postsynaptic potential (PSP) responses to the first and second stimuli of the train showed an apparent reduction in presynaptic release probability after CCI (see Supplemental Results and Discussion, Reduction in presynaptic release probability in neurons of CCI mice, and Supplemental Figure 1).

In summary, a congruent picture emerged of substantial reductions in intrinsic and synaptic neuronal activity and spontaneous and sensory-evoked local network excitability 48 hours after TBI, at a time point that coincided with neuronal edema mediated by a change in NKCC1/KCC2 expression. We next asked whether the edema and excitability changes were linked.

The CCC inhibitor bumetanide reduces neuronal swelling and membrane capacitance 48 hours after CCI. We tested whether the increased NKCC1/KCC2 ratio responsible for the neuronal swelling 48 hours after injury was also responsible for the changes in excitability. We administered the CCC inhibitor bumetanide at $2 \mathrm{mg} /$ $\mathrm{kg}$, a dose that should be selective for NKCC1 inhibition $(54,55)$, 30 minutes before CCI $(39,56)$. Confirming our initial results, vehicle-treated CCI mice injected with synapsin 1-driven GCaMP6f (to visualize neurons) had an increase in neuronal cross-sectional area compared with sham-treated mice. In contrast, bumetanide-treated CCI neurons had a smaller cross-sectional area, similar to that of sham-treated neurons $(P=0.001,1$-way ANOVA with Bonferroni's multiple-comparisons test; CCI-bumetanide vs. vehicle: $P<$ 0.001, CCI-vehicle vs. sham-vehicle: $P<0.01 ; n=54-64$ neurons, $n=5-6$ mice per group) (Figure $4 \mathrm{~A}$ ).

To assess whether bumetanide was acting through peripheral mechanisms (e.g., diuretic effects) (55), we tested chlorothiazide (CTZ) $(10 \mathrm{mg} / \mathrm{kg}$, i.p.) (57), which lacks effects on central NKCC1 cotransporters (acting instead on kidney cotransporters) (55). In contrast to bumetanide, CTZ had no effect on neuronal surface area $(P=0.001$, Bonferroni's multiple-comparisons test; CCIvehicle vs. sham-vehicle: $P<0.05$ and CCI-CTZ vs. sham-CTZ: $P$ $<0.01 ; n=49-66$ neurons, $n=3-5$ mice per group) (Supplemental Figure 2). Thus, the neuronal swelling we observed appeared to be dependent on NKCC1 expressed in the CNS.

Interestingly, the capacitance of CCI-bumetanide-treated neurons was smaller than that of neurons from CCI vehicle-treated mice and similar to that of sham-treated neurons $(P=0.0009$, Bonferroni's multiple-comparisons test; CCI-bumetanide vs. vehicle: $P=0.002$, CCI-vehicle vs. sham-vehicle: $P=0.001 ; n=$ 8-10 neurons, $n=6-8$ mice per group) (Figure $4 \mathrm{~B}$ ). This is what would be expected if membrane capacitance closely followed the membrane surface area and, other factors being equal, predicts a membrane excitability increase with bumetanide treatment.

Bumetanide treatment increases intrinsic and network excitability in CCI neurons. The decreased $R_{\text {in }}$ we initially observed in neurons after CCI was replicated in CCI vehicle-treated animals. This decreased $R_{\text {in }}$ was reversed by bumetanide ( $P=0.01$, Bonferroni's multiple-comparisons test; CCI-bumetanide vs. vehicle: $P=$ $0.004, \mathrm{CCI}-\mathrm{vehicle}$ vs. sham-vehicle: $P=0.03 ; n=8-10$ neurons, $n=6-8$ mice per group) (Figure $4 \mathrm{C}$ ). We observed an increase in $\mathrm{AP}$ frequency in response to depolarizing current steps in CCIbumetanide-treated neurons compared with CCI-vehicle-treated neurons (Figure 4D), resulting in a significantly increased I-V curve slope $(P=0.01$, Bonferroni's multiple-comparisons test; 
A

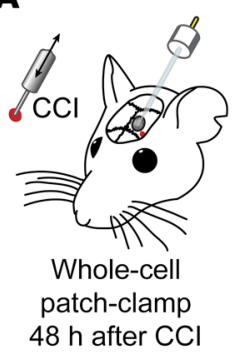

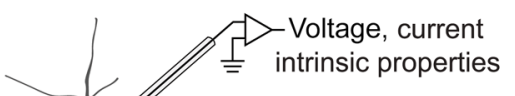

intrinsic properties
Frequency of action potentials $(\mathrm{Hz})$

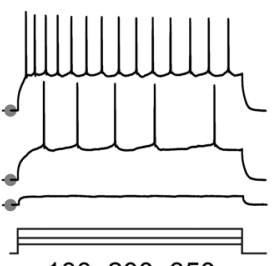

$100,200,350$ input currents $(\mathrm{pA})$

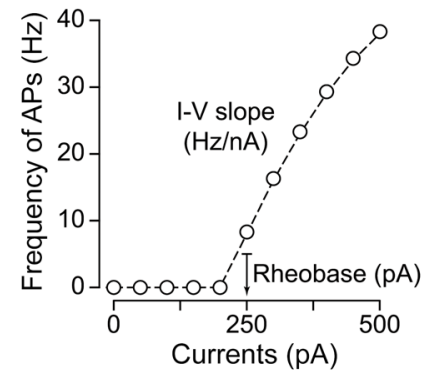

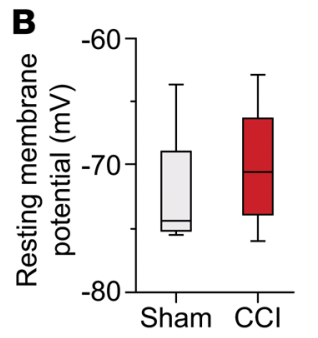

F

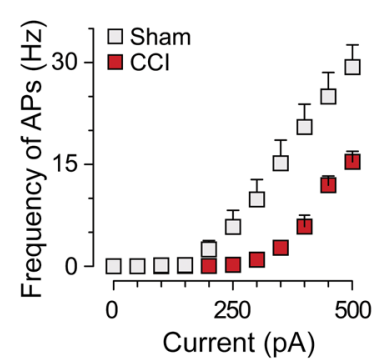

C

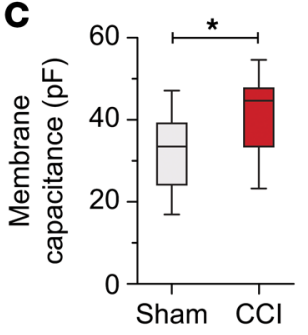

G

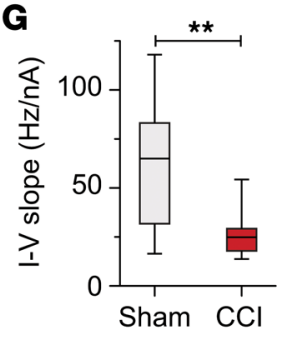

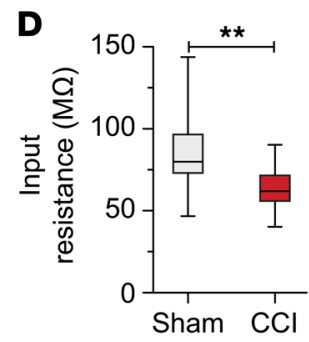

H

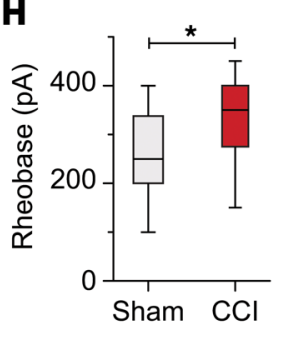

E
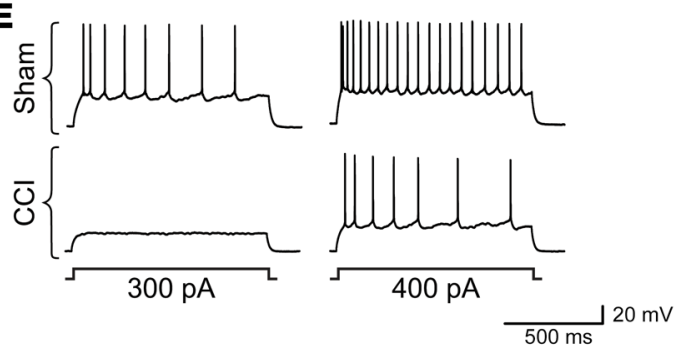

I

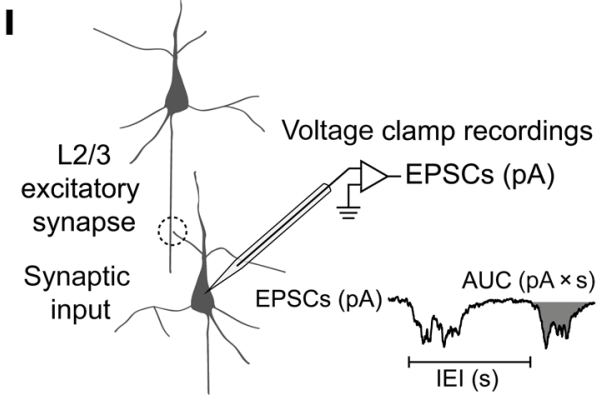

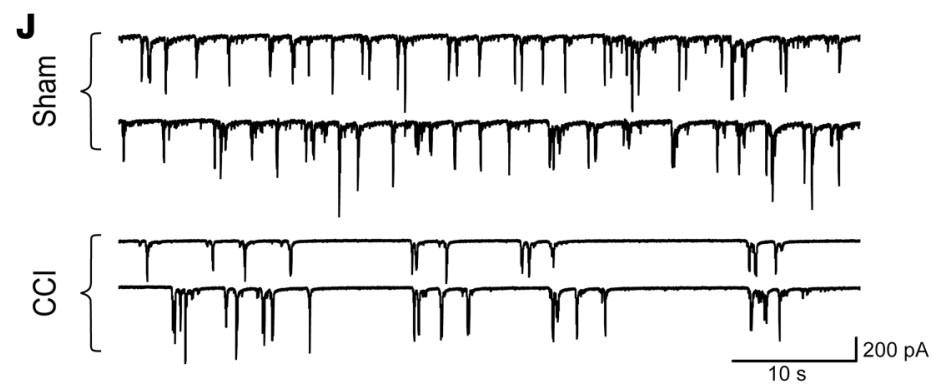
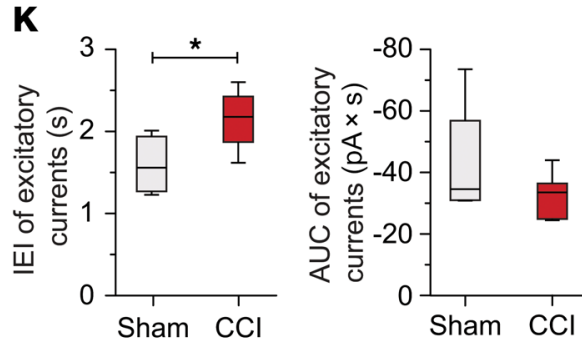

Figure 2. Decreases in neuronal intrinsic excitability and excitatory currents $\mathbf{4 8}$ hours after CCI. (A) Schematic shows in vivo whole-cell recording methods, and parameters used to characterize the intrinsic properties of the neuronal soma. Changes in intrinsic membrane properties are known to modify the response property of a cell. (B) No difference in RMP was detected between sham and CCI treatment groups $(P>0.05,2$-sided, unpaired $t$ test; $n=9$ - 10 neurons, $n=6-10$ mice per group). (C) Box-and-whisker plot showing increased membrane capacitance in neurons of CCI-treated mice $\left({ }^{*} P=0.01\right.$, unpaired $t$ test; $n=14-15$ neurons, $n=10-12$ mice per group). (D) After CCI, the mean $R$ of layer $2 / 3$ pyramidal cells was significantly decreased $\left({ }^{* *} P=0.007\right.$, Mann-Whitney $U$ test; $n=14-15$ neurons, $n=10-12$ mice per group). (E) Typical whole-cell recordings from pyramidal neurons showing AP firing at 300 and 400 pA currents for sham- and CCI-treated mice. Scale bar: 20 mV, 500 ms. There was reduced AP firing in CCI neurons at each current amplitude. (F) Plot of mean firing frequency as a function of current intensity. Note the decreased firing in the CCI-treated neuron relative to the sham-treated neuron ( $n=$ 12-13 neurons, $n=7-11$ mice per group). (C) I-V curve showed a significant decrease in slope for neurons from CCI-treated animals (** $P=0.001$, MannWhitney $U$ test; $n=12-13$ neurons, $n=7-11$ mice per group). (H) CCI neurons showed increased rheobase ( ${ }^{*} P=0.04$, unpaired $t$ test; $n=12-13$ neurons, $n=$ 7-11 mice per group). (I) Schematics show voltage-clamp recordings of incoming excitatory synaptic information that neurons receive across their dendritic arbors. (J) Typical voltage-clamp recordings show spontaneous inward excitatory currents in layer 2/3 pyramidal cells of the sensory cortex of sham- and CCI-treated mice. Scale bar: 200 pA, 10 seconds. (K) Mean IEls were longer (frequency was decreased) in cells from CCI-treated animals $\left({ }^{*} P=0.03,2\right.$-sided Mann-Whitney $U$ test; $n=6-7$ neurons, $n=6-7$ mice per group). No differences in the AUC of excitatory currents were observed between groups (right plot: $P>0.05$, 2-sided Mann-Whitney $U$ test; $n=6-7$ neurons, $n=6-7$ mice per group).

CCI-bumetanide vs. vehicle: $P=0.005$, CCI-vehicle vs. shamvehicle: $P=0.01$ ) (Figure $4 \mathrm{E}$ ). CCI-bumetanide-treated neurons also had a lower rheobase compared with CCI vehicle-treated neurons $(P=0.005$, Bonferroni's multiple-comparisons test;
CCI-bumetanide vs. vehicle: $P=0.001, \mathrm{CCI}$ vehicle vs. sham vehicle: $P=0.02$ ) (Figure $4 \mathrm{~F}$ ). These results show that blockade of NKCC1/KCC2 ratio-dependent neuronal edema was associated with increases in intrinsic membrane excitability after TBI and, 
A

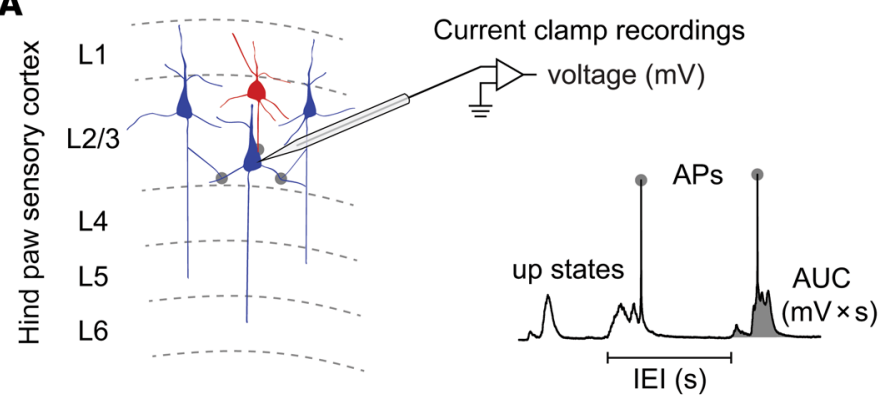

B

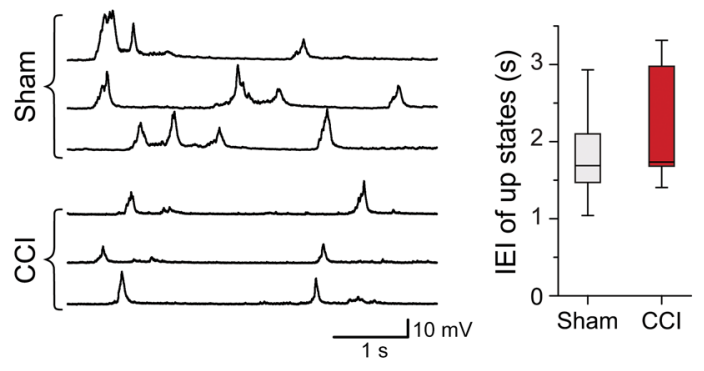

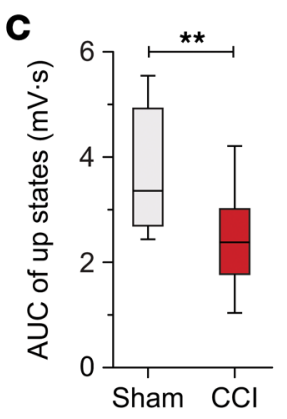

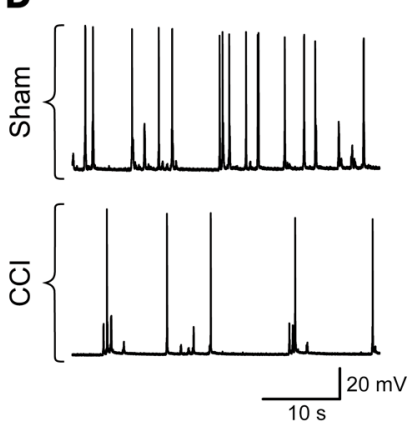

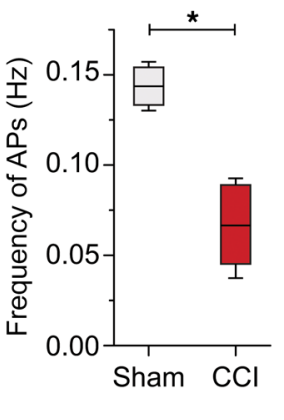

E Whole-cell patch-clamp, $48 \mathrm{~h}$ after $\mathrm{CCl}$

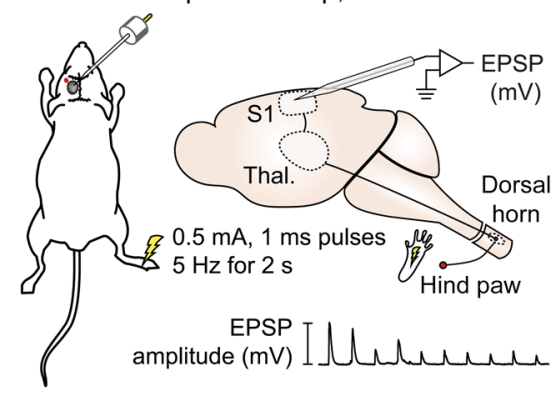

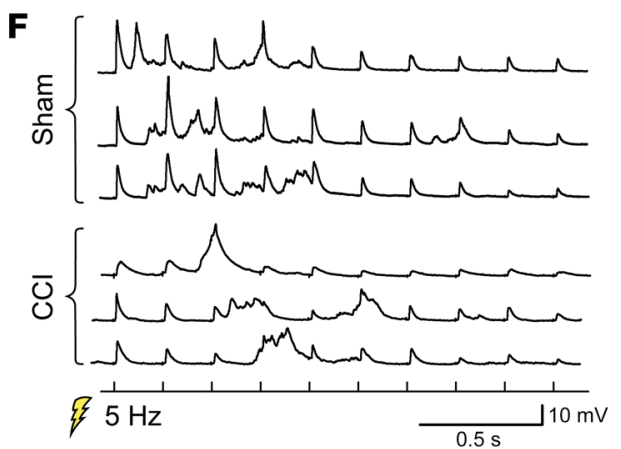

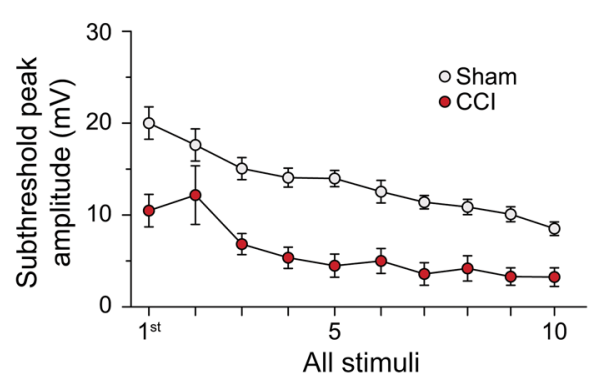

All stimuli

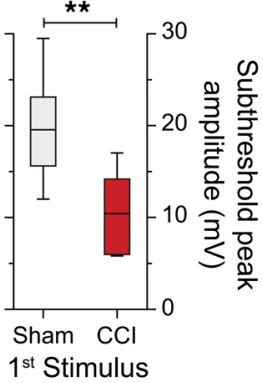

Figure 3. Reduced network activity and APs in somatosensory cortex $\mathbf{4 8}$ hours after CCI. (A) Schematics of intracellular current-clamp recordings measuring up states (subthreshold local network events) and APs. (B) Representative traces of spontaneous membrane potential fluctuations from layer $2 / 3$ pyramidal neurons. Scale bar: $10 \mathrm{mV}, 1$ second. Average up state IEI box-and-whisker plot showing no difference in frequency after injury $(P>0.05,2$-sided, unpaired $t$ test; $n=12$ neurons, $n=8-10$ mice per group). (C) Plot of the mean AUC of up states showing a smaller area in the neurons of mice that underwent CCI ( ${ }^{* *} P=0.007,2$-sided, unpaired $t$ test; $n=12$ neurons, $n=8-10$ mice per group). (D) Traces of AP firing. Scale bar: $20 \mathrm{mV}, 10$ seconds. Box-andwhisker plot shows a lower frequency of spontaneous APs in the neurons of CCI-treated mice ${ }^{*} P=0.01$, 2-sided Mann-Whitney $U$ test; $n=4-5$ neurons, $n=4-5$ mice per group). (E) Schematic of the experimental design for in vivo whole-cell sensory stimulation experiments. Evoked activity was recorded in layer 2/3 of the somatosensory cortex (hind limb area). Thal., thalamus. (F) Typical traces of subthreshold responses evoked by contralateral hind paw stimulation at the RMP. Scale bar: $10 \mathrm{mV}, 0.5$ seconds. The population subthreshold voltage response is plotted as a function of the stimulus number ( $P<0.0001$, 2-way ANOVA; $n=6-9$ neurons, $n=6-8$ mice per group). We observed a decrease in amplitude for all responses in CCI neurons during a $5 \mathrm{~Hz}$, 2 second train of hind paw stimuli. The plot on the right shows a response to first sensory stimuli in neurons of CCI-treated mice ${ }^{* *} P=0.003$, unpaired $t$ test; $n=6-9$ neurons, $n=6-8$ mice per group).

therefore, that CCC-dependent neuronal edema was associated with a reduction in excitability.

To determine whether CCC-dependent neuronal edema contributed to reduced synaptic excitability, we examined spontaneous excitatory currents with in vivo voltage-clamp recordings. Neurons from CCI-bumetanide-treated mice showed a significant decrease in EPSC IEIs (increased frequency) relative to neurons from CCI-vehicle-treated mice $(P=0.005$, Kruskal-Wallis with Dunn's multiple-comparisons test; CCI-bumetanide vs. vehicle: $P$ $<0.01$, CCI-vehicle vs. sham-vehicle: $P>0.05 ; n=7-9$ neurons, $n=$ 5-8 mice per group) (Figure $4, \mathrm{G}$ and $\mathrm{H}$ ). CCI-bumetanide-treated neurons also had a larger EPSC AUC relative to CCI-vehicle-treated neurons $(P=0.01$, Kruskal-Wallis with Dunn's multiple-comparisons test; CCI-bumetanide vs. vehicle: $P<0.05$, CCI-vehicle vs. sham-vehicle: $P<0.05$ ) (Figure $4, \mathrm{G}$ and $\mathrm{H}$ ).

Next, using in vivo current clamp recordings, we examined the local network activity revealed by up states after bumetanide treatment. Though we observed no change in the frequency of up states between groups (IEI $P>0.05$, Kruskal-Wallis with Dunn's multiple-comparisons test; $n=6-10$ neurons, $n=5-10$ mice per group) (Figure 5, A and B), up states in CCI-bumetanide-treated neurons had a significantly larger AUC compared with that of up 
A
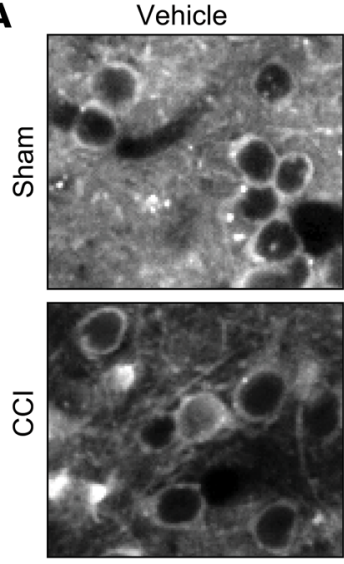

Bumetanide
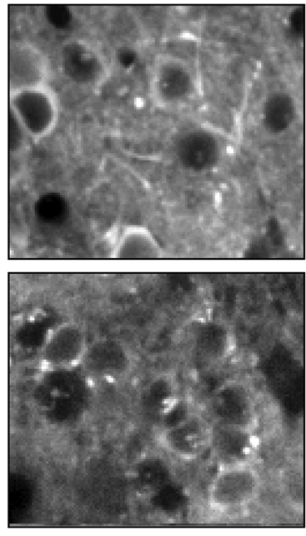

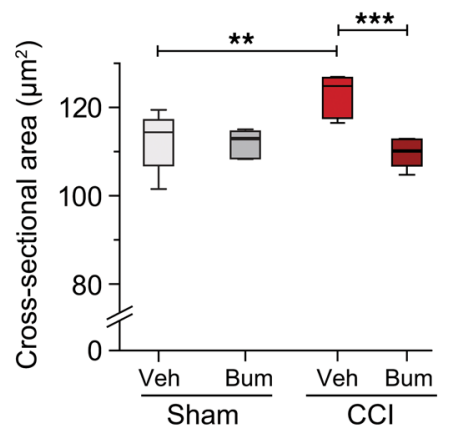

B

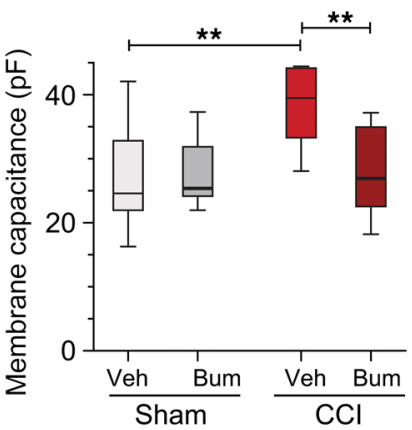

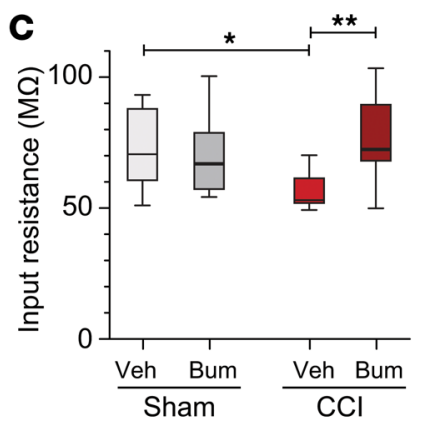

D

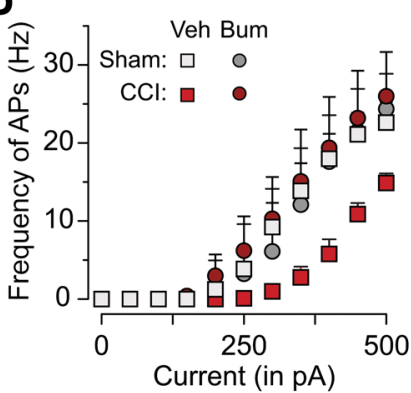

E

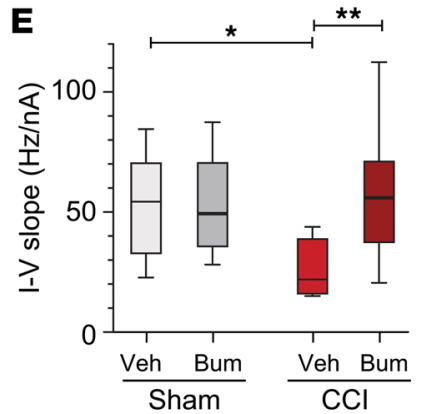

$\mathbf{F}$

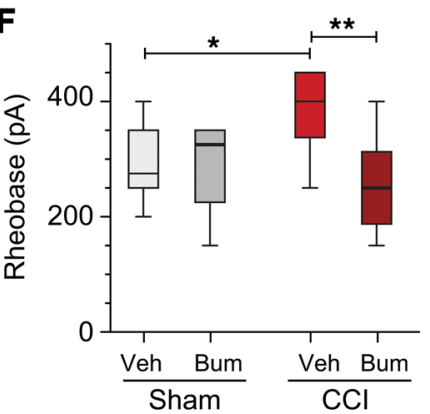

G
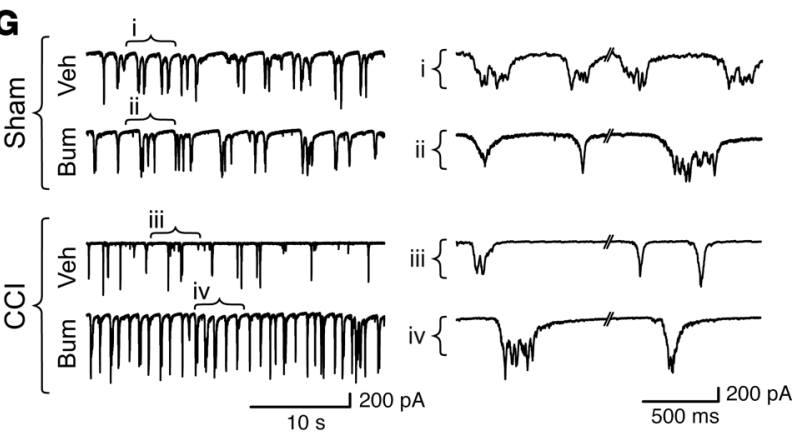

H

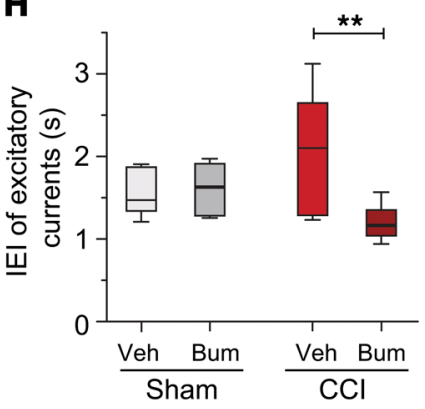

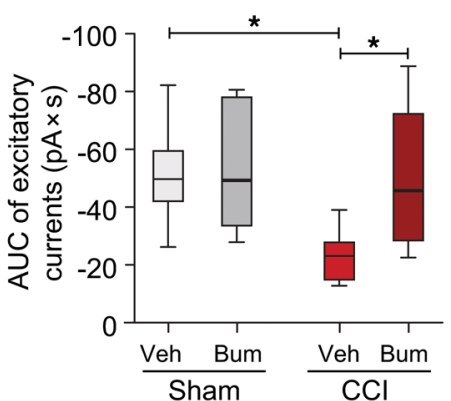

Figure 4. The CCC inhibitor bumetanide eliminates neuronal swelling and increases neuronal intrinsic and excitatory activity. (A) Images for each group. Scale bar: $20 \mu \mathrm{m}$. Quantification of neuronal cross-sectional area showed a reversal of edema with bumetanide (Bum) treatment $(P=0.001,1$-way ANOVA with Bonferroni's multiple-comparisons test; CCI-bumetanide vs. vehicle [Veh]: ${ }^{* *} P<0.001$; CCI-vehicle vs. sham-vehicle: ${ }^{* *} P<0.01 ; n=54-64$ neurons, $n=5-6$ mice per group). (B) Consistent with a reversal of edema, membrane capacitance was reduced in CCI-bumetanide neurons compared with CCIvehicle neurons $\left(P=0.0009\right.$, Bonferroni's multiple-comparisons test; $C \mathrm{Cl}$-bumetanide vs. vehicle: ${ }^{* *} P=0.002$; CCI vehicle vs. sham-vehicle: ${ }^{* *} P=0.001$; $n=8-10$ neurons, $n=6-8$ mice per group).The following membrane properties were measured: (C) input resistance $(P=0.01$, Bonferroni's multiple-comparisons test; CCl-bumetanide vs. vehicle: ${ }^{* *} P=0.004$; CCI-vehicle vs. sham-vehicle: ${ }^{*} P=0.03 ; n=8-10$ neurons, $n=6-8$ mice per group); (D) plot of the mean firing frequency as a function of current intensity ( $n=8-10$ neurons, $n=6-8$ mice per group); (E) I-V slope ( $P=0.01$, Bonferroni's multiple-comparisons test; $\mathrm{CCl}$-bumetanide vs. vehicle: ${ }^{*} P=0.005$; CCI-vehicle vs. sham-vehicle: ${ }^{*} P=0.01 ; n=8-10$ neurons, $n=6-8$ mice per group); and $(\mathbf{F})$ rheobase $(P=$ 0.005 , Bonferroni's multiple-comparisons test; CCI-bumetanide vs. vehicle: ${ }^{*} P=0.001$; CCI-vehicle vs. sham-vehicle: ${ }^{*} P=0.02 ; n=8-10$ neurons, $n=6-8$ mice per group). These measurements showed increases in intrinsic membrane excitability in CCI-bumetanide neurons compared with CCI-vehicle neurons. (G) Typical recordings of spontaneous excitatory currents. Scale bar: 200 pA, 10 seconds. Traces on the right show expanded currents (i-iv). Scale bar: 200 pA, 500 ms. (H) IEls were shorter (frequencies were higher) in the CCI-bumetanide-treated group compared with the CCl-vehicle-treated group $(P=0.005$, Kruskal-Wallis with Dunn's multiple-comparisons test; CCI-bumetanide vs. vehicle: ${ }^{*} P<0.01$; CCI-vehicle vs. sham-vehicle: $P>0.05 ; n=7-9$ cells, $n=$ 5-8 mice per group). Box-and-whisker plot on the right shows a larger AUC of excitatory currents in CCI-bumetanide neurons $(P=0.01$, Kruskal-Wallis with Dunn's multiple-comparisons test; CCI-bumetanide vs. vehicle: ${ }^{*} P<0.05$; CCI-vehicle vs. sham-vehicle: ${ }^{*} P<0.05 ; n=7-9$ cells, $n=5-8$ mice per group).

states in CCI-vehicle-treated neurons ( $P=0.001$, Kruskal-Wallis with Dunn's multiple-comparisons test; CCI-bumetanide vs. vehicle: $P<0.05$, CCI-vehicle vs. sham-vehicle: $P<0.001$ ) (Figure 5 , $A$ and B). Finally, we compared the responses to sensory stimulation after bumetanide treatment. Neurons from CCI-bumetanide- treated mice showed increased EPSP amplitude during trains of 5 $\mathrm{Hz}$ stimuli compared with CCI-vehicle-treated neurons $(P<0.05$, 2-way ANOVA test with Bonferroni's multiple-comparisons test; CCI-bumetanide vs. vehicle: $P<0.0001$, CCI-vehicle vs. shamvehicle: $P<0.0001 ; n=4-6$ neurons, $n=4-6$ mice per group) (Fig- 
A
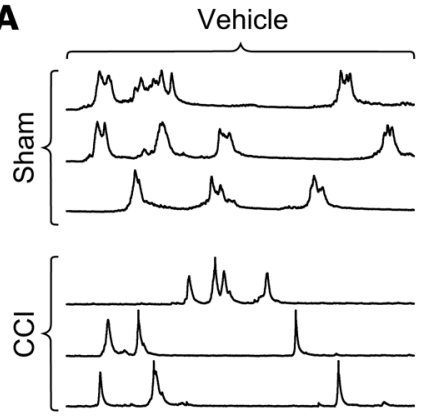

C
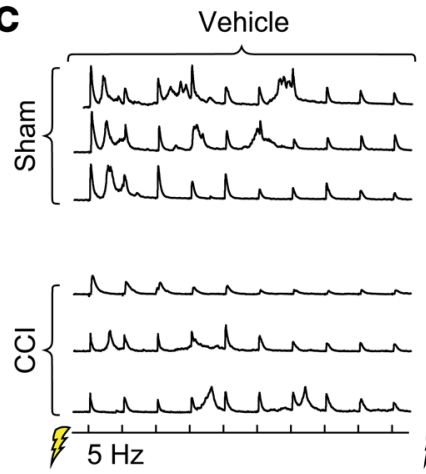
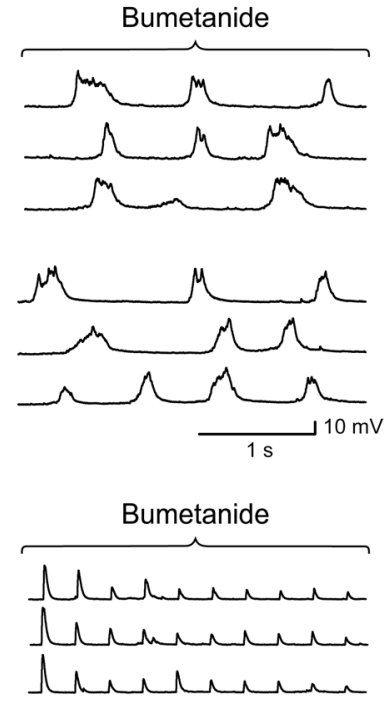

denMmara

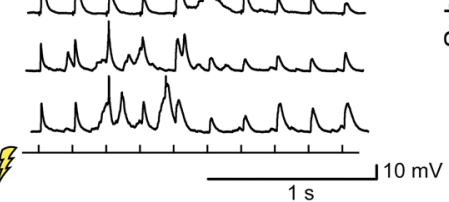

B
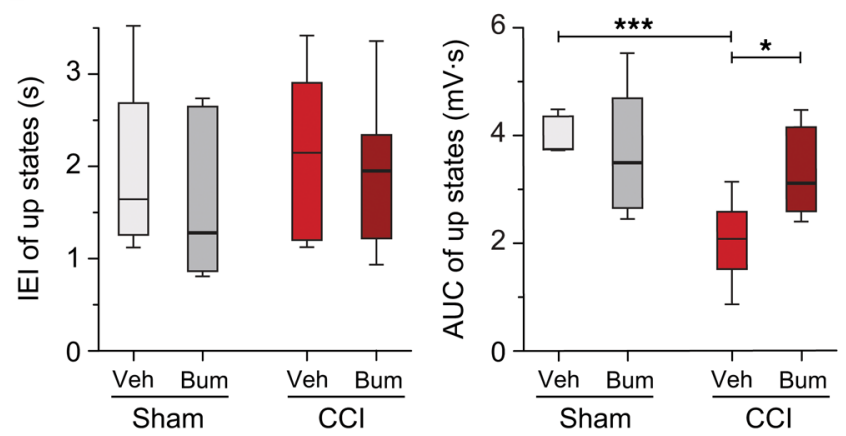

D

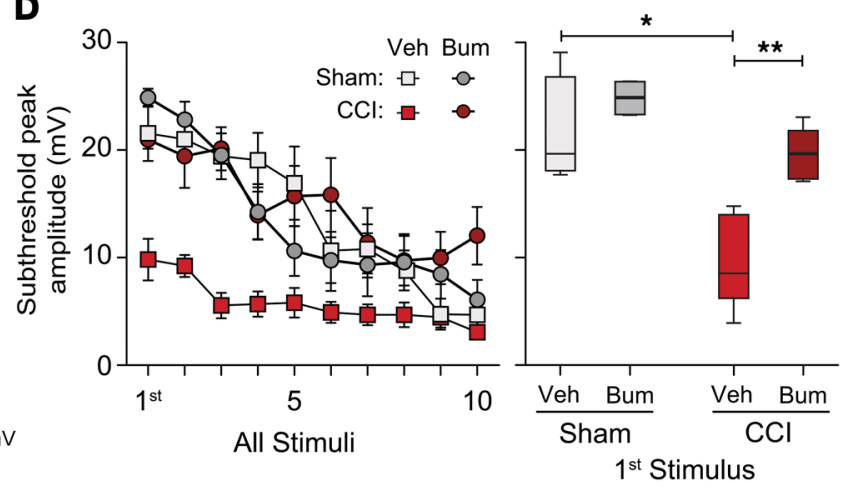

Figure 5. Reducing neuronal edema increases network excitability after CCI. (A) Representative traces of up states. Scale bar: 10 mV, 1 second. (B) No changes in IEls of up states were observed between groups $(P>0.05$, Kruskal-Wallis with Dunn's multiple-comparisons test; $n=6-10$ neurons, $n=5-10$ mice per group). The AUC of up states was larger in the CCI-bumetanide-treated group than in the CCI-vehicle-treated group ( $P=0.001$, Kruskal-Wallis with Dunn's multiple-comparisons test; CCI-bumetanide vs. vehicle: ${ }^{*} P<0.05$; CCI-vehicle vs. sham-vehicle: ${ }^{* *} P<0.001$ ). (C) Traces showing typical subthreshold responses to $5 \mathrm{~Hz}$ hind paw stimulation. Scale bar: $10 \mathrm{mV}$, 1 second. (D) CCI-bumetanide treatment increased EPSP responses to subsequent trains of $5 \mathrm{~Hz}$ stimuli, relative to $\mathrm{CCI}$ neurons $(P<0.05,2$-way ANOVA test with Bonferroni's multiple-comparisons test; $C \mathrm{CI}$-bumetanide vs. vehicle: $P<$ $0.0001, \mathrm{CCl}$-vehicle vs. sham-vehicle: $P<0.0001 ; n=4-6$ neurons, $n=4-6$ mice per group). Plot on right shows larger sensory-evoked voltages (first EPSP in a train of stimuli) in neurons from CCI-bumetanide mice $\left(P=0.003\right.$, Kruskal-Wallis test; CCI-bumetanide vs. vehicle: ${ }^{*} P<0.01$; CCI-vehicle vs. shamvehicle: ${ }^{*} P<0.05 ; n=4-6$ neurons, $n=4-6$ mice per group).

ure 5, C and D). This strongly suggests that bumetanide-suppressible, CCC-dependent neuronal edema is responsible for the reductions in local and sensory-induced network activity after CCI.

Bumetanide treatment increases susceptibility to SDs and seizures 48 hours after CCI. SDs provide a metric of net cortical excitability (13), but more important, they have been definitively associated with TBI in humans and are correlated with worsened outcomes $(11,12,27)$. We observed a spontaneous SD in each animal immediately following CCI (within 30 seconds of impact) (see Supplemental Results and Discussion, Spontaneous spreading depolarization after CCI, and Supplemental Figure 3). We next measured SD susceptibility in response to a continuous potassium chloride stimulus (58) in sham- and CCI-treated mice that were administered bumetanide or vehicle 48 hours after CCI (Figure 6, A and B). We detected no statistically significant difference in the number of SDs between sham- and CCI-vehicle-treated animals (Figure 6, C and $\mathrm{D}$ ). Making the more clinically relevant comparison between vehicle- and bumetanide-treated animals after CCI, we observed a statistically significant increase in the number of SDs in CCIbumetanide-treated animals. This was true for both "full" SDs, which were observed to propagate across the imaging field, and "partial" SDs, which were smaller events that did not propagate fully (full SDs: $P=0.04$, Kruskal-Wallis with Dunn's multiple-com- parisons test; CCI-bumetanide vs. vehicle: $P<0.05$; Figure 6, B and C; total [full plus partial] SDs: $P=0.03$; Kruskal-Wallis with Dunn's multiple-comparisons test; CCI-bumetanide vs. vehicle: $P<0.05 ; n=7-9$ mice per group; Figure 6, B and D).

Like SDs, seizures are a major and debilitating consequence of TBI $(12,17)$ and represent a hyperexcitable network state. In order to evaluate the potential of neuronal edema to affect seizure susceptibility, we used the minimal clonic seizure test in vivo. This assay was selected because of its utility as a putative forebrain or cortical seizure model $(59,60)$. We observed no change in the percentage of animals with seizures between sham- and CCI-vehicle-treated animals (Figure 7). However, when comparing CCI-vehicle and CCI-bumetanide groups, the bumetanide-treated group showed a higher percentage of animals with seizures compared with CCI-vehicle-treated animals (CCI-bumetanide: $55.55 \%$, CCI-vehicle: 0\%, sham vehicle: $10 \%$, sham-bumetanide: $44.44 \% ; \chi^{2}$ test; CCI-bumetanide vs. vehicle: $P=0.004 ; n=9-10$ mice per group) (Figure 7).

In summary, we observed increases in susceptibility to the 2 primary forms of network hyperexcitability that follow TBI SD and seizures - after treating neuronal edema with the CCC inhibitor bumetanide.

Mannitol reduces neuronal edema and increases susceptibility to SDs and seizures. Next, we wanted to test the relationship 
A
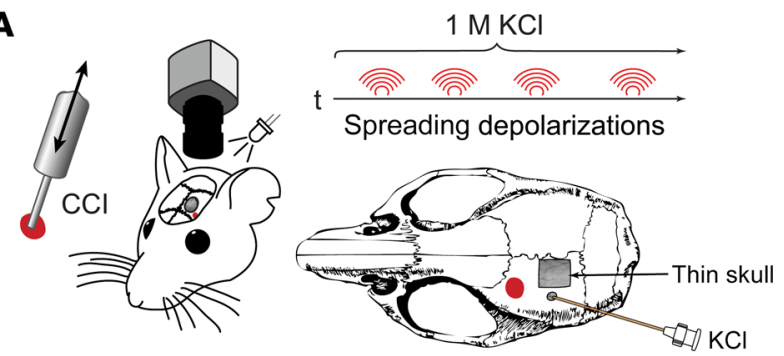

B
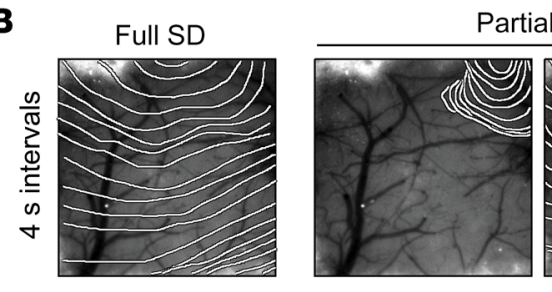

Partial SD
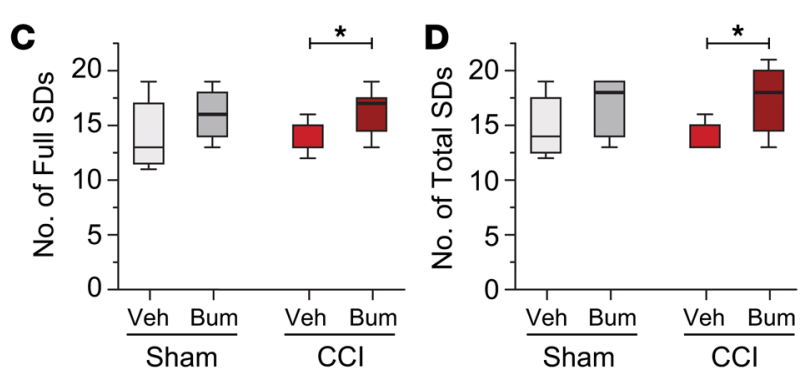

between edema and neuronal function using a clinically deployed drug. Mannitol is an osmotic agent that is a first-line treatment for intracranial pressure elevation associated with brain edema (61). In addition to removing free water by osmotic diuresis, the drug is also shown to mediate decreases in neuronal volume and intraneuronal chloride through CCCs, at least in the developing brain (62). Animals received a single dose of mannitol in saline (3 $\mathrm{g} / \mathrm{kg}$, i.v.) (63) thirty minutes before trauma, and the control mice were injected with saline.

Neuronal edema in mice injected with synapsin 1-driven GCaMP6f (32) was reduced following treatment with mannitol 48 hours after injury. The neuronal cross-sectional area was smaller in CCI-mannitol-treated mice compared with that in CCI-vehicletreated mice and was similar to that in sham mannitol- and sham vehicle-treated animals $(P=0.001$, 1-way ANOVA with Bonferroni's multiple-comparisons test; CCI-mannitol vs. vehicle: $P<$ 0.01 , CCI-vehicle vs. sham-vehicle: $P<0.01 ; n=32-70$ neurons, $n=4-5$ mice per group) (Figure $8 \mathrm{~A}$ ).

We tested susceptibility to SDs and seizures, with the prediction that these would be increased with reduced neuronal edema. CCI-mannitol-treated mice showed an increased SD frequency compared with CCI vehicle-treated mice $(P=0.03$, KruskalWallis with Dunn's multiple-comparisons test; $\mathrm{CCI}$ mannitol vs. vehicle: $P<0.01 ; n=6-10$ mice per group) (Figure $8 \mathrm{~B})$. Similarly, CCI-mannitol-treated animals showed a higher percentage of seizures compared with CCI-vehicle-treated mice (CCI-mannitol: $62.50 \%$, CCI-vehicle: $22.22 \%$, sham-vehicle: $66.66 \%$, sham-mannitol: $42.85 \%$; CCI-mannitol vs. vehicle: $P=0.04$, CCI-vehicle vs. sham-vehicle: $P=0.02, \chi^{2}$ test; $n=7-9$ mice per group) (Figure $8 \mathrm{C}$ ).
Figure 6. Increases in susceptibility to SDs with reductions in neuronal edema. (A) Schematic shows the experimental design for SD susceptibility experiments. (B) Representative images show changes in the intrinsic signal under white light during passage of full and partial SD waves. Contours are drawn every 4 seconds at the wavefront of SDs. Scale bar: 0.5 $\mathrm{mm}$. Analysis of full (C) and total (D) SDs (including partial SDs) showed a significant increase in the number of SDs in the CCI-bumetanidetreated group relative to the CCI-vehicle-treated group (full SDs: $P=0.04$, Kruskal-Wallis with Dunn's multiple-comparisons test; CCI-bumetanide vs. vehicle: ${ }^{*} P<0.05$ and total SDs: $P=0.03$, Kruskal-Wallis with Dunn's multiple-comparisons test; CCI-bumetanide vs. vehicle: ${ }^{*} P<0.05 ; n=7-9$ mice per group).

Taken together, these results indicate that mannitol, a drug commonly used in clinical practice, increases SD and seizure susceptibility with a concurrent reduction in neuronal volume, 48 hours after TBI. The mannitol results showed that a reduction in neuronal volume by any means (rather than exclusively via the NKCC1/KCC2 ratio) can generate increases in excitability. Finally, this is the first demonstration to our knowledge that mannitol reduces neuronal edema after TBI in vivo.

Increased susceptibility to SDs 1 week after CCI. We next examined the longer-term effects of neuronal volume on excitability. We noted (Figure 1, E and H) that 1 week after CCI, despite a small but statistically significant reduction in KCC2 expression, neuronal volume had normalized, and we asked whether this was associated with an increase in excitability. We observed a significant increase in SD numbers in CCI- versus sham-treated mice ( $P=$ 0.04 ; 2-sided, unpaired $t$ test; $n=11-12$ mice per group) (Figure 9). These 1-week data add another layer of confirmation that normalized neuronal volume is associated with increased excitability after TBI, and bring up the possibility of spontaneous increases in excitable events as edema recedes.

\section{Discussion}

We applied new tools - in vivo whole-cell recording combined with 2-photon microscopy - to study the network consequences of edema after TBI (Figure 10). Our primary finding was that neuronal edema, which we definitively confirmed occurred after TBI, was associated with an unexpected reduction in excitability. This reduction was likely due to the effects of neuronal swelling, which results in increased membrane capacitance and decreased resistance. Interestingly, neuronal excitability was reduced despite changes in NKCC1/KCC2 cotransporter expression that would be expected to have increased excitability (see below). Both the swelling and the excitability decrement were reversible with the CCC inhibitor bumetanide, showing that the neuronal volume and excitability changes were linked and identifying the molecular mechanism of the neuronal swelling.

Importantly, the blockade of neuronal swelling with bumetanide also increases the incidence of SDs and seizures, two network excitability events that are associated with worsened outcomes in brain injury $(11,12,27)$. SD and seizure susceptibility were also increased when we treated neuronal edema with mannitol, an osmotic agent commonly used in clinical practice. Finally, 1 week after TBI, when edema had resolved spontaneously, we observed an increase in the incidence of SDs. Overall, our data demonstrate that neuronal swelling exerts a protective effect against excitable 

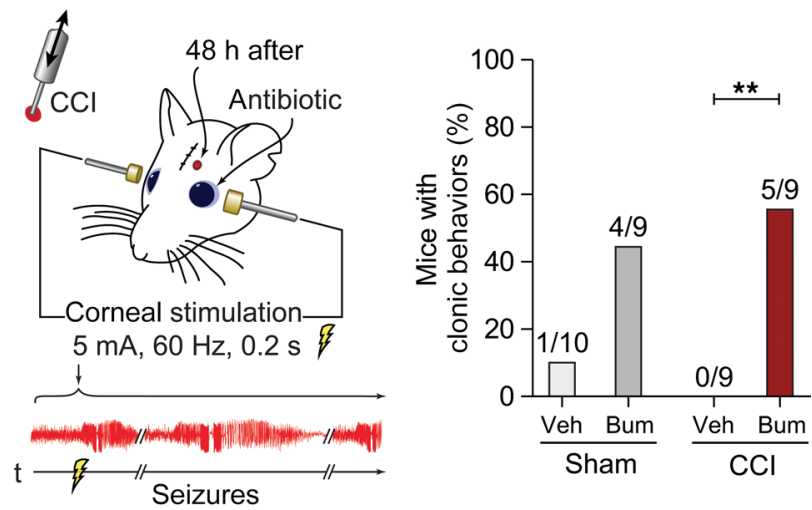

Figure 7. Blockade of edema with bumetanide increases seizure susceptibility in vivo. Schematic of a seizure behavioral model. Graph shows the percentage of animals with seizure activity in sham- and CCl-treated mice treated with vehicle or bumetanide. A higher percentage of animals had seizures in the $\mathrm{CCl}$-bumetanide-treated group than in the $\mathrm{CCl}$-vehicletreated group ( ${ }^{* *} P=0.004, \chi^{2}$ test; $n=9-10$ mice per group; the number of mice with seizures and group size are indicated for each group.).

events in the early aftermath of TBI and that treatment of edema may increase the risks of these events.

Neuronal edema in the aftermath of TBI. Astrocytic swelling after TBI has been well documented $(36,64,65)$ and is considered a manifestation of the cytotoxic edema that follows injury. In contrast, the neuronal volume response after TBI is relatively uncharacterized $(5,6)$. It is noteworthy that only 1 study explicitly measured neuronal swelling after TBI using Nissl staining ex vivo (6). Our findings show that neuronal edema does occur in the cortex in vivo after TBI (Figure 1D, Figure 4A, Figure 8A, Supplemental Figure 2, Supplemental Figure 4A, and Supplemental 5A). Additionally, our combined protein expression (Figure 1, F and G) and pharmacological (Figure 4A and Supplemental Figure 4A) findings show that the mechanism of neuronal edema, at least in part, is an increase in the NKCC1/KCC2 ratio (Supplemental Figure 7).

CCC roles in TBI-associated edema. CCCs play a crucial role in the maintenance of neuronal volume via the regulation of ionic and water homeostasis $(37,66)$. The NKCC1 cotransporter is an electroneutral transporter of chloride into the cell, and it moves water in the same direction as ions to counteract osmotic changes (590 water molecules for $\left.1 \mathrm{Na}^{+}, 1 \mathrm{~K}^{+}, 2 \mathrm{Cl}^{-}\right)(37,67)$. The KCC2 cotransporter pumps chloride out of the neuron, causing a net water efflux and neuronal shrinkage (500 water molecules for $1 \mathrm{~K}^{+}$and $\left.1 \mathrm{Cl}^{-}\right)(37,67)$. A change in the $\mathrm{NKCC} 1 / \mathrm{KCC} 2$ ratio can be due to upregulation of NKCC1 expression $(38,39)$, downregulation of KCC2 expression (38), or both. In each case, the result is an increase in neuronal intracellular water content. We observed a decrease in KCC2 expression levels as early as 2 hours after injury (but see refs. 68, 69), and this decrease persisted 48 hours and 1 week after TBI, similar to data from prior studies (refs. 68, 69 and Figure 1, F-H). We observed no change in NKCC1 protein expression from 2 hours to 1 week after TBI $(56,69,70)$. Others have observed increases in NKCC1 expression 2 hours after TBI $(56,70)$, which contrasts with our data but would be expected to lead to the same outcome of increased neuronal volume.
Prior pharmacological data also support a role for CCCs in brain edema (see Supplemental Results and Discussion, Role of $S D$-associated neuronal edema in the response to TBI). Bumetanide treatment reduced TBI-induced bulk brain edema (quantified as a change in the percentage of brain water content) and astrocytic swelling (see Supplemental Results and Discussion, Possible effects of bumetanide on astrocytic CCCs), thus showing relevance to multiple cellular edema mechanisms. This effect is thought to be due to changes in protein expression, specifically the inhibition of NKCC1 upregulation observed after injury $(39,56)$. Because the half-life of bumetanide is short (47 minutes in mice; ref. 71) and the drug was dosed only once, cotransporter binding by the drug itself is less likely to be the mechanism (see also Supplemental Results and Discussion, Bumetanide effects on network excitability 48 hours after CCI are likely due to changes in cotransporter gene expression). In our study, the findings were somewhat different, but the theme is similar. As shown in prior work, we found that bumetanide probably operated through changes in gene expression, because treatment 30 minutes before and immediately after CCI prevented edema at 48 hours (Figure $4 \mathrm{~A}$ and Supplemental Figure 4A), but treatment at the 48-hour time point (a point at which transporter binding rather than expression changes would be expected; Supplemental Figure 5A) was ineffective. In contrast to prior work, instead of preventing a rise in NKCC1 expression, we found that bumetanide reversed a reduction in KCC2 protein expression (Supplemental Figure 6A), an effect that would also be predicted to reduce edema, and indeed did (Figure 4A). A similar reversal of KCC2 downregulation has been observed with bumetanide treatment in epilepsy models (72). Interestingly, bumetanide treatment in our hands was also associated with an increase in NKCC1 expression (Supplemental Figure 6B), which would be expected to have an opposite effect, i.e., an increase in neuronal volume. We speculate that NKCC1 expression changes may represent a compensatory response to a primary effect of bumetanide on KCC2 expression; however, without better temporal resolution of the protein expression (and potentially phosphorylation), this cannot be confirmed.

More broadly, differences in data on CCC expression and the effects of bumetanide after TBI may have multiple causes: differences in the species studied (rats [refs. 56, 68, 70] vs. mice [refs. 39, 69]); edema measurements (bulk tissue edema [refs. 39, 56, 70] vs. astrocytic swelling [ref. 73] vs. neuronal swelling); TBI methods (weight drop [refs. 56, 70] vs. CCI without craniotomy [ref. 69] vs. CCI [ref. 68]); TBI severity with the same CCI method (39); brain regions (choroid plexus; [ref. 56] vs. hippocampus [ref. 70] vs. cortex [refs. 39, 68, 69]); and time points for expression analysis (bumetanide treatment: 8 hours [ref. 56], 24 hours [ref. 39] vs. 2 hours; NKCC1: 1 hour, 24 hours, 5 days [ref. 39] vs. 2 hours, 48 hours, 7 days; KCC2: 3 hours, 24 hours, 7 days [ref. 68] vs. 2 hours, 48 hours, 7 days), among others. However, there is consistency in the overall results, suggesting a role for CCC activity in both neuronal and bulk tissue edema, with the ratio of NKCC1/ KCC2 expression being the key variable, and a role for CCC blockers in edema suppression.

Competing effects of volume and chloride content on neuronal excitability. Both neuronal volume (41-43) and chloride content (38) can affect excitability, but they do so in opposite directions 
A
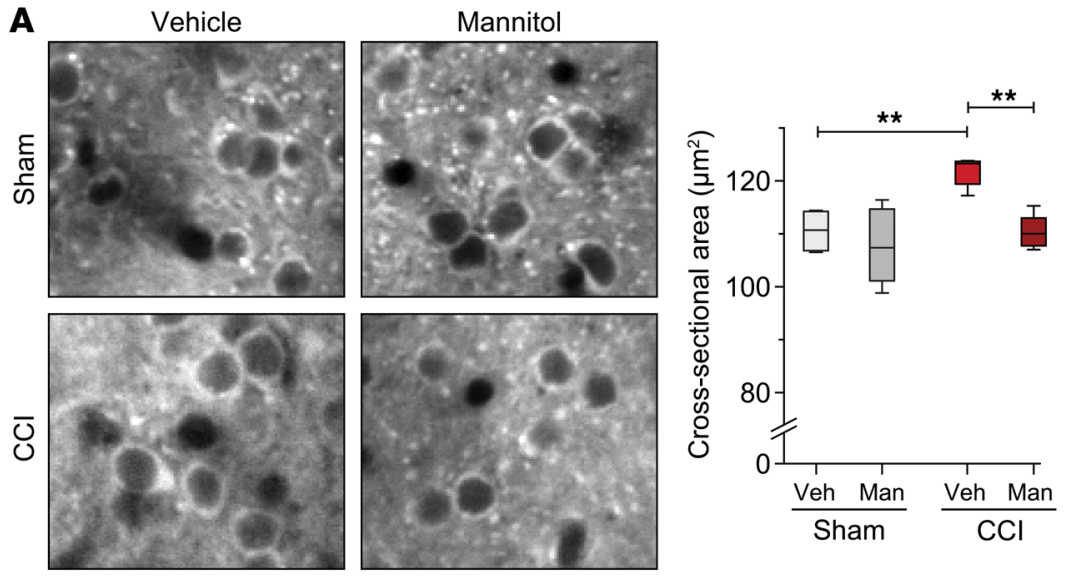

B

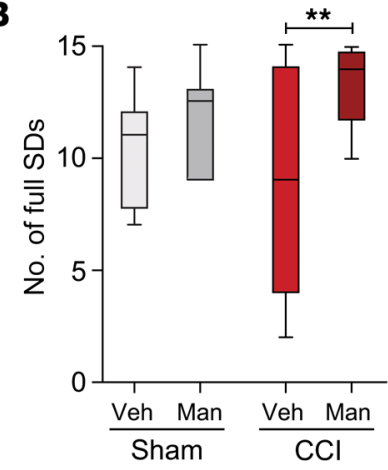

C

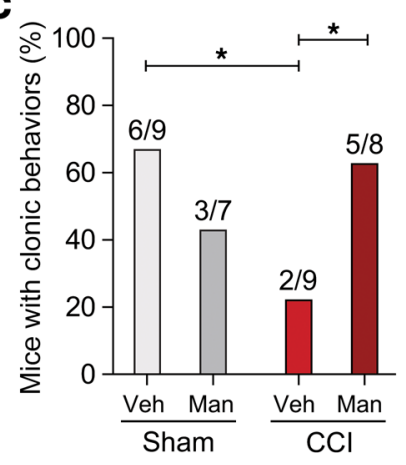

Figure 8. The osmotic diuretic mannitol reduces neuronal swelling and increases network activity. (A) Images of representative neurons in layer $2 / 3$ cortex during treatments. Scale bar: $25 \mu \mathrm{m}$. Plot of neuronal cross sectional area shows that mannitol (Man) $(3 \mathrm{~g} / \mathrm{kg}$; i.v.) reversed neuronal swelling in CCI-treated mice $(P=$ 0.001, 1-way ANOVA with Bonferroni's multiple-comparisons test; $\mathrm{CCl}$-mannitol vs. vehicle: ${ }^{* *} P<0.01$; CCI-vehicle vs. sham-vehicle: ${ }^{*} P<0.01 ; n=32-70$ neurons, $n=4-5$ mice per group). (B) Quantification of SD frequency based on 2-hour recordings in $1 \mathrm{M} \mathrm{KCl}$ solution showed a significant increase in SD susceptibility in CCI-mannitol-versus CCI-vehicle-treated animals $(P=$ 0.03 , Kruskal-Wallis with Dunn's multiple-comparisons test; CCI-mannitol vs. vehicle: ${ }^{*} P<0.01 ; n=6-10$ mice per group). (C) Analysis of network excitability showed a significant increase in the percentage of animals with seizures in the CCI-mannitol-treated group relative to the CCI-vehicle-treated group (CCI-mannitol vs. vehicle: ${ }^{*} P=0.04 ;$ CCl-vehicle vs. sham-vehicle: ${ }^{*} P=0.02 ; \chi^{2}$ test; $n=7-9$ mice per group; the number of mice with seizures and group size are indicated for each group.).
(Figure 10). As neuronal volume increases, membrane surface area, and thus membrane capacitance, increase (41-43). Though resistance and capacitance are not necessarily dependent on each other, increases in capacitance will, other factors being equal, result in decreased input resistance (41-43). A cell with decreased input resistance is less excitable, as depolarizing currents have smaller effects on membrane potential. However, the increased NKCC1/KCC2 ratio responsible for neuronal swelling is also associated with increased intracellular chloride levels $(40,66)$. Higher intracellular $\mathrm{Cl}^{-}$concentrations $\left(\left[\mathrm{Cl}^{-}\right]_{\mathrm{i}}\right)$ would be predicted to generate a more depolarized chloride equilibrium potential, decreasing and potentially reversing the driving force for chloride entry during GABA receptor activation (66). This can lead to reduced efficacy of inhibition, shunting inhibition, or even an excitatory effect of GABA receptor activation $(40,66)$. Thus, while pure membrane biophysics considerations would predict decreased excitability with neuronal swelling, the chloride handling associated with that swelling would predict increased excitability. Our data show that the increased capacitance and decreased resistance associated with neuronal edema dominate over altered chloride-mediated excitability, leading to decreased neuronal and network excitability 48 hours after CCI.

$S D$ and seizure susceptibility after CCI and edema treatment. Our observation of reductions in intrinsic, synaptic, local network, and sensory-driven activity 48 hours after TBI (Figures 2 and 3) suggested a robust and consistent change from the neuronal membrane to a distributed network level. We wanted to know whether this change in network activity had consequences that might be translationally relevant. SDs and seizures are the most common secondary brain insults following TBI $(14,15)$. Although SDs are more prevalent than seizures in human recordings after brain injury (53\% SD prevalence vs. $~ 15 \%$ seizure prevalence) $(17,74,75)$, both have the potential to exacerbate tissue damage $(13,25,26)$, and both occur together in animal models (19-24) and in humans after TBI (17). Importantly, both SDs and seizures in patients with TBI are correlated with poorer clinical outcomes $(14,27)$ and are being considered as measurable biomarkers of injury (76).

Comparing the susceptibility of animals exposed to TBI, we found that both bumetanide, which directly targets edema mechanisms, and mannitol, which is in clinical use, suppressed edema but at the same time were associated with substantial increases in $\mathrm{SD}$ and seizure susceptibility compared with vehicle treatment (Figure 4A, Figures 6-8, and Supplemental Figure 4). This strongly suggests that edema exerts a protective effect, at least at the 48-hour time point after TBI, via the reduction of SD and seizure susceptibility. We observed a statistically significant reduction in seizure susceptibility after CCI (Figure 8C), consistent with this hypothesis (see Supplemental Results and Discussion, Mechanisms of SD and seizure susceptibility after TBI). Interestingly, we did not observe a similar reduction in SD susceptibility. However, the SD experiments were not powered to detect a difference in SD numbers of less than approximately $25 \%$, so we may have missed a smaller difference. Given the convergence of intrinsic, synaptic, local network, and seizure data on an excitability reduction after CCI, we suspect that the SD data may represent a false negative. Overall, the comparison of the treated and untreated CCI condition (rather than the comparison of sham with CCI treatment considered here) is arguably the most relevant to clinical practice, 


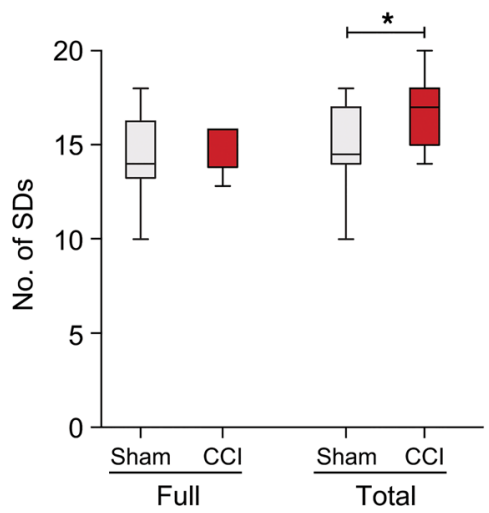

Figure 9. Increased susceptibility to SDs 1 week after $\mathrm{CCI}$, with no neuronal swelling but mildly altered chloride cotransporter expression. $A$ significant increase in the total number of SDs (full and partial SDs) was seen in CCl-treated mice relative to sham-treated mice, after neuronal swelling had spontaneously resolved 1 week following $\mathrm{CCl}\left({ }^{*} P=0.04\right.$; 2-sided, unpaired $t$ test; $n=11-12$ mice per group).

where the injury is a nonnegotiable aspect of patient care, and the choices come at the level of treatment options.

Importantly, though with slightly different mechanistic considerations, SD susceptibility data gathered 1 week after CCI also support the link between neuronal volume and excitability, as resolved neuronal swelling was associated with an increased susceptibility to SDs (Figure 1E and Figure 9). Interestingly, this occurred despite a small but still statistically significant reduction in KCC2 expression that might have been expected to be associated with an increase in neuronal volume. It is possible that, at this time point, other volume regulation mechanisms (e.g., impermeant anions) (77) compensated for the effects of reduced KCC2 expression. Alternatively, it is possible that there is a threshold below which KCC2 changes are not sufficient to exert volume changes, or that our detection methods were not sensitive enough to detect small changes. Whatever the explanation, the phenotype we observed was one of increased excitability, which we attribute to an increase in intracellular chloride unopposed by membrane swelling. More specifically, with decreased KCC2 expression, a neuron has less GABAergic inhibition, as reduced chloride extrusion causes an increase in intracellular chloride and a depolarizing shift in $\mathrm{GABA}_{\mathrm{A}}$ reversal potential $(40,66)$. This agrees with prior in vitro work that showed a depolarizing shift in $\mathrm{GABA}_{\mathrm{A}}$ reversal potential (with an increase in $\left[\mathrm{Cl}^{-}\right]_{\mathrm{i}}$ ) associated with a reduction in KCC2 protein and mRNA expression in the dentate gyrus (78). Our data suggest that, in addition to edema treatment, the spontaneous resolution of neuronal edema may open a "window of excitability" mediated by inefficient inhibitory neurotransmission unopposed by volume changes. In the case of edema resolution, this "window" could occur regardless of treatment strategy.

Translational implications. The implications of a neuroprotective effect of edema after TBI are clinically relevant; thus, it is imperative that they be considered very carefully. Most important, our findings need to be interpreted within the temporal dynamics of recovery from injury. We have shown a potentially protective reduction in excitability associated with edema between 2 and 48 hours after injury that appears to end with an increase in excitabil- ity with edema resolution by 1 week after injury. However, altogether different conditions may prevail at different time points or in areas distant from the injury (see also Supplemental Results and Discussion, Spatial profile of CCI-associated changes in excitability). It is noteworthy that, although cerebral edema, measured by intracranial pressure (ICP) elevation, is associated with worse clinical outcomes, treatment of elevated ICP has not been shown to substantially improve outcomes $(4,79)$. In heterogeneous patient populations, this finding may have many explanations, but a hypothesis based on our work is that this lack of substantial improvement may at least in part be due to increased excitability associated with edema treatment. The primary translational implication of our work is not that edema needs to be completely reconsidered as a harmful factor after TBI, but that the edema/excitability dynamic after brain injury is more complex than previously understood.

\section{Methods}

Animals. Adult male C57BL6/J mice (The Jackson Laboratory, stock no. 000664; used for electrophysiology, Western blot, seizure behavioral, and SD experiments); synapsin 1-driven GCaMP6f-injected C57BL6/J mice (32) (AAV1.Syn.GCaMP6f.WPRE.SV-40, Addgene, no. 100837; used for 2-photon neuronal cell size measurements with bumetanide, mannitol, and CTZ treatments); and Thy1-GCaMP6s mice (32) [C57BL/6J-Tg(Thy1-GCaMP6s)GP4.3Dkim; The Jackson Laboratory, stock no. 024275; used for neuronal cell size experiments with bumetanide injected immediately and 48 hours after CCI] were used in this study. All mice were 2 months old and had a mean weight of $25.4 \mathrm{~g}$. Animals were randomly assigned to the experimental groups. The experimenter was blinded to the groups analyzed in the electrophysiological, SD counting, seizure behavior, and histology experiments. Analysis of electrophysiological, 2-photon cell size, and cell counting data was performed in a blinded manner. The number of replicates is mentioned with each data set.

CCI. Bumetanide (2 mg/kg, MilliporeSigma, B3023) (57) (in vehicle, $0.5 \mathrm{~N} \mathrm{NaOH}$ in saline, i.p.) or mannitol $(3 \mathrm{~g} / \mathrm{kg}$, in saline, i.v., MilliporeSigma, M4125) (63) were delivered 30 minutes before CCI or sham surgery. Animals were anesthetized with isoflurane $(1.5 \%)$ in a $70 \% / 30 \%$ nitrogen/oxygen mixture, and vital signs were monitored and maintained within a physiologically normal range (temperature: $36.5^{\circ} \mathrm{C}-37.5^{\circ} \mathrm{C}$; heart rate: $450-550 \mathrm{bpm} ; \mathrm{SpO}_{2}: 92 \%-95 \%$ ). We made a $1.5 \mathrm{~mm}$ craniotomy $3 \mathrm{~mm}$ lateral to the midline at Bregma using a dental drill (Figure 1A). TBI was induced on the exposed cortex using a controlled impactor device (Leica Biosystems) with a 1 $\mathrm{mm}$ diameter tip, a velocity of $4 \mathrm{~m} / \mathrm{s}$, a depth of $1.0 \mathrm{~mm}$, and a dwell time of $100 \mathrm{~ms}$ to produce "moderate" CCI injury (cortical but no observable hippocampal damage) (81). After surgery, the animals were placed on a heating blanket until they regained mobility. The sham-operated animals underwent identical anesthesia, craniotomy, and recovery procedures but no CCI. The animals were placed in their home cage for 48 hours of recovery before electrophysiology, imaging, or histology experiments.

In vivo whole-cell recordings. We used in vivo patch-clamp techniques to record from layer $2 / 3$ neurons in the mouse somatosensory cortex, similar to our previously published methods (46). Mice were anesthetized using urethane (1.5 g/ kg, i.p.) (82) supplemented with isoflurane $(\sim 0.5 \%)$, if necessary. The depth of anesthesia was assured by continuous monitoring of the breathing rate, which was more than 
A

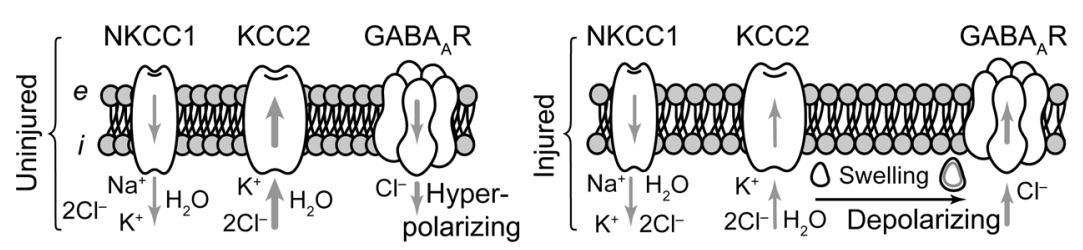

B

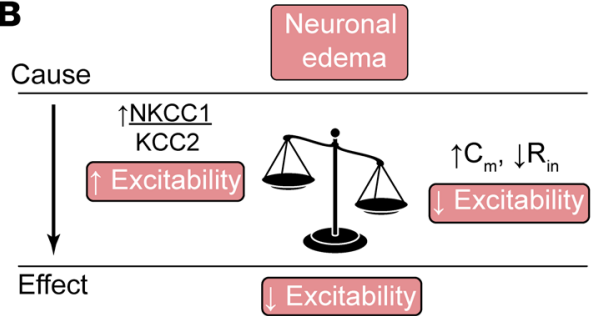

C

Cell
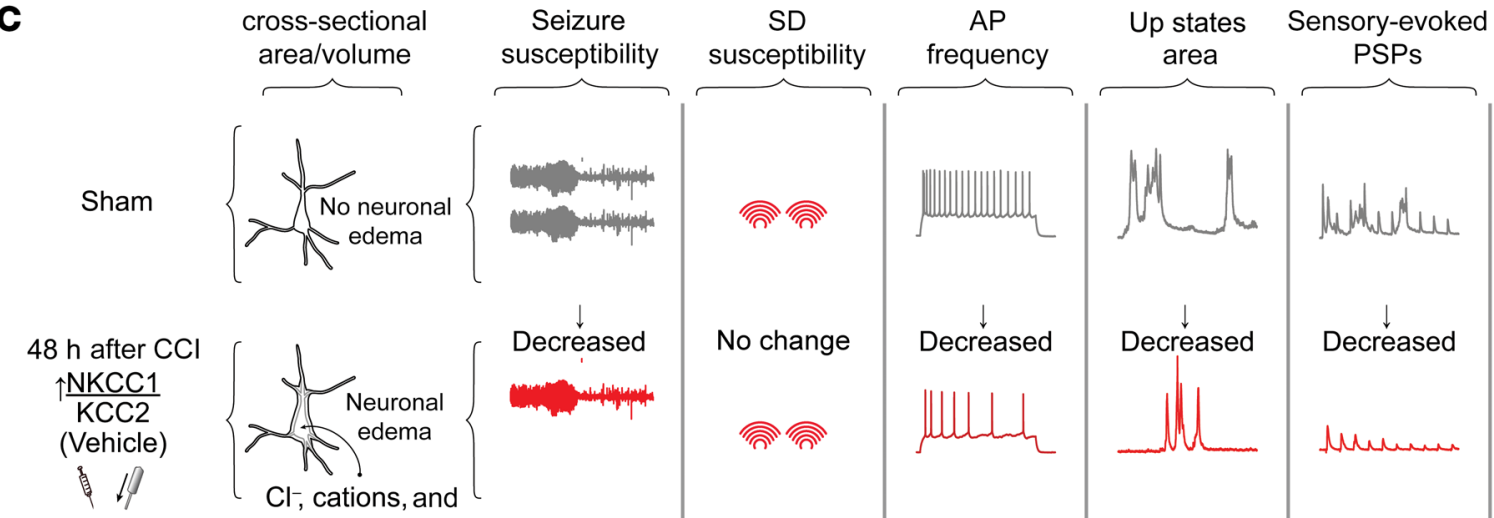

Excitatory
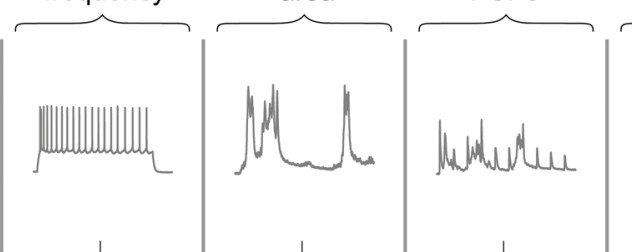
currents
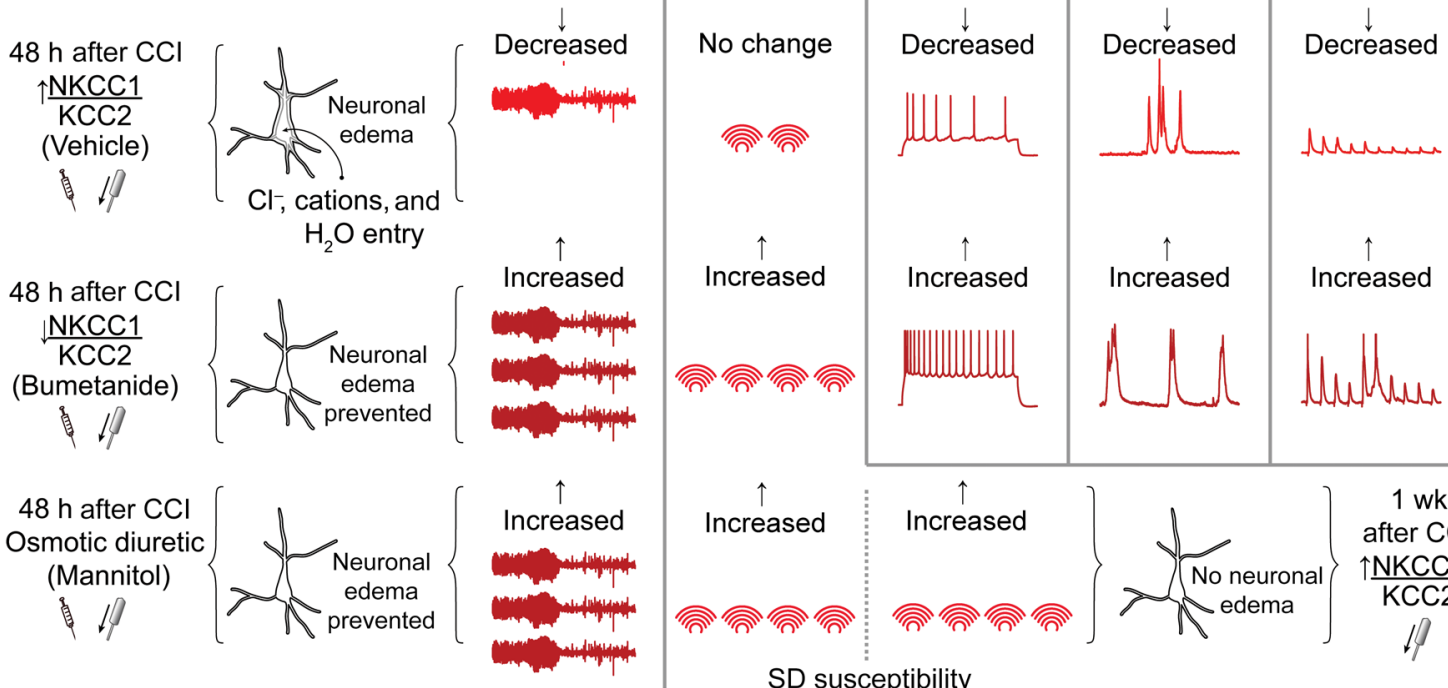

Decreased

SD susceptibility

Figure 10. Edema and neuronal excitability after TBI. (A) Schematic shows neuronal membrane with proteins known to be active in both neuronal volume and excitability (NKCC1, KCC2, and GABA ${ }_{A}$ receptors) before and after CCI, with predicted changes in ionic flux and membrane diameter (cell size). (B) Cell swelling and chloride fluxes should have opposite effects on excitability, with swelling predicted to be associated with a reduction in excitability and swelling-associated chloride shifts with an increase in excitability. Schematic shows the observed effects, with cell volume effects prevailing over chloride effects. Dep., depolarizing. (c) Summary of experimental findings.

90 breaths per minute. This was maintained by altering the isoflurane level. Additionally, to maintain a consistent depth of anesthesia, recordings were started when up states occurred at approximately $1 \mathrm{~Hz}$ frequency (83). Neuronal recordings were made approximately $2.0 \mathrm{~mm}$ from the center of the CCI lesion (Figure 1, A and B). Briefly, we used patch electrodes of 3 to $4 \mathrm{M} \Omega$ resistance that were filled with intracellular solution containing $135 \mathrm{mM}$ K-gluconate, $8 \mathrm{mM} \mathrm{NaCl}, 5$ mM EGTA, 10 mM HEPES, 0.3 mM GTP, 2 mM ATP, 7 mM phosphocreatine ( $\mathrm{pH}$ 7.2), and an osmolality of approximately $295 \mathrm{mOsm}$. In current-clamp configuration, recordings were made at the cells' RMP $(-71.58 \pm 1.08 \mathrm{mV}$; range: -63.78 to $-75.84 \mathrm{mV})$. Voltage-clamp experiments were performed with a potassium gluconate internal solution at a clamped potential of $-70 \mathrm{mV}$.

Signals were amplified, digitized at $10 \mathrm{kHz}$, and stored for analysis as previously described (46). If access resistance changed more than $20 \%$, the recording was excluded from analysis. We examined the neuronal I-V curve, which was determined by measuring the frequen- cy of AP firing in response to stepped current injections $(-50$ to +500 $\mathrm{pA}, 1000 \mathrm{~ms}$ square pulses). $R_{\text {in }}$ was measured in voltage-clamp at -70 $\mathrm{mV}$ from the steady-state current in response to $-20 \mathrm{mV}$ voltage steps. Rheobase was the smallest current evoking an AP. Amplitude, width, AUC, and IEI were characterized using the first 50 events.

In vivo 2-photon imaging. Two-photon images were captured using a Sutter Movable Objective Microscope with 2 Hamamatsu R6357 photomultiplier tubes, a Zeiss 20×/1.0 NA water immersion objective, and a Spectra-Physics MaiTai Ti:Sapphire laser with an excitation pulse width of $100 \mathrm{fs}$, a wavelength of $920 \mathrm{~nm}$, and an emission of 535/50 $\mathrm{nm}$. Laser power at the objective was less than $50 \mathrm{~mW}$. Images were acquired at $2.96 \mathrm{~Hz}$ and $256 \times 256$ pixel resolution using ScanImage software (84). High-magnification images (optical: $20 \times$, digital: $2 \times$; resolution $0.75 \mu \mathrm{m} /$ pixel $)$ were acquired from a $192 \times 192 \times 200 \mu \mathrm{m}(1 \mu \mathrm{m}$ vertical step) volume of cortical tissue, starting from the pia. Targeting layer $2 / 3$, maximum-intensity projections were used to find the maximum diameter of all cells within 130 to $190 \mu \mathrm{m}$ of the $200 \mu \mathrm{m} Z$-stack. 
Imaging was performed from 3 fields of view per mouse (all in the hind paw somatosensory cortex), with the average cross-sectional area computed from all neurons within these 3 regions ( $>10$ cells per area).

Intrinsic signal imaging. Male C57BL/6J mice were used to avoid sex-linked effects on SD susceptibility (85). Imaging was performed following our previously published methods (58). Briefly, the cortex was illuminated via a white light-emitting diode, and intrinsic signal was collected using a MiCAM02 high-sensitivity monochrome charge-coupled device (CCD) camera (SciMedia; $696 \times 520$ pixels, exposure $20 \mathrm{~ms}, 0.5 \mathrm{~Hz}$ ). Offline analysis of SD transients was made with Image J software (NIH) $(58,86)$. A plot of the $z$-axis profile was used to count SD totals over the 2-hour period. SDs were grouped into 2 categories: full and partial. A partial SD designation was assigned to wave fronts that did not propagate over the entirety of the cortex in the field of view (Figure 6B).

Minimal clonic seizure model. Focal clonic seizures were induced in mice via corneal stimulation using custom-made corneal electrodes (brass eye cups soldered to stainless steel wires) and an electroconvulsometer (ref. 60 and Figure 7). Following stimulation, the animals exhibited seizures including forelimb clonus, jaw clonus, and head nodding. We first evaluated the convulsive current that would elicit seizures in a naive population of animals ( $n=10$ mice), using $60 \mathrm{~Hz}$ of alternating current, with stimulus intensities of 5 to $15 \mathrm{~mA}$ delivered over 0.2 seconds. Using a Probit analysis, we determined the current intensity that would elicit seizures in approximately $25 \%$ of the test population $(\mathrm{CC} 25=5 \mathrm{~mA})$. This convulsive current was selected because it would allow for detection of the increase in seizure susceptibility that was hypothesized for brain-injured animals treated with bumetanide. Group sizes for seizure behavioral testing $(n=10)$ were selected, because this would allow for detection of an increase of $20 \%$ (90\% power, $\chi^{2}$ test, $\alpha$ level of 0.05 ).

Hind paw stimulation and evoked responses. Sensory responses were evoked with electrical stimulation through custom-made needles placed into the contralateral hind paw pad. One-millisecond electrical stimuli were delivered at $0.5 \mathrm{~mA}$ intensity at $5 \mathrm{~Hz}$ for a total of 10 stimuli over 2 seconds (87). Layer $2 / 3$ pyramidal neurons in vivo show sparse firing characteristics $(46,88)$. Thus, evoked APs were not analyzed because of the low number of events per trial per animal. Peak amplitude of subthreshold EPSPs was measured from the baseline to the peak of the synaptic depolarization.

Fluoro-Jade B staining. Following perfusion and postfixation 48 hours after CCI, $30 \mu \mathrm{m}$ thick coronal sections were cut using a tissue slicer and mounted. Sections were collected from bregma +2.0 to $-3.0 \mathrm{~mm}$. Fluoro-Jade B (FJB) staining (MilliporeSigma AG310) was performed following previously published methods (89). The number of FJB-positive neurons in the cortex was determined by blinded quantitative histological analysis (https://www.unige.ch/ medecine/bioimaging/files/3714/1208/5964/CellCounting.pdf). After thresholding and binarization, cells were counted automatically from images in ImageJ (86). Clusters were separated into individual cells using the Watershed function in ImageJ. Neuronal counts were determined on 5 sections separated by $250 \mu \mathrm{m}$, at a magnification of $400 \times$. Cell numbers were normalized to the area of staining to yield the number of cells per area. Those values were averaged for all sections for each animal.

Western blot analysis. Western blotting was performed in a manner similar to our previously published methods (57). Protein samples were incubated with polyclonal antibodies against KCC2 (1:1000, MilliporeSigma, catalog 07-432) or NKCC1 (1:500, MilliporeSigma, catalog AB3560P), or with a monoclonal $\beta$-tubulin antibody (1:10,000, MilliporeSigma, catalog T5201) at room temperature for 2 hours. The blots were then incubated with HRP-conjugated anti-rabbit IgG (1:2000, GE Healthcare, catalog NA934) or anti-mouse antibodies (1:2000, GE Healthcare, catalog NA931) for 2 hours at room temperature. Blots were visualized with ECL (Amersham).

Statistics. Data were analyzed using Minitab 19 Statistical Software (Minitab), MATLAB (version 7.8.0), and GraphPad Prism (version 5.00 for Windows, GraphPad Software). Statistical box plots show the following: median (horizontal line), 25th to 75th percentile range (box), and 5th to 95th percentile range (whiskers). Both meanand median-based measures of central tendency are presented to provide a complete characterization of the data. In the supplemental materials, we have presented the distribution of all data as minimum, first quartile (25th percentile), median, third quartile (75th percentile), maximum, and mean $( \pm$ SEM) values. Outliers were excluded using Grubb's test. A parametric $t$ test and ANOVA were used when the data sets were found to be normally distributed, with homogeneity of variance determine by the Shapiro-Wilk test or Bartlett's and Levene's tests, respectively, otherwise a Mann-Whitney $U$ test and KruskalWallis with Dunn's multiple-comparisons test were used, with a significance cutoff of 0.05. To obtain the differences among animals showing seizure behaviors, the $\chi^{2}$ test was applied. Data are represented as percentages. For the data in all figures, statistical significance was set at ${ }^{*} P<0.05,{ }^{* *} P<0.01,{ }^{* *} P<0.001$, and ${ }^{* * *} P<0.0001$.

Study approval. Experimental procedures were performed with the approval of the IACUC of the University of Utah and in accordance with Animal Research: Reporting of In Vivo Experiments (ARRIVE) criteria (80).

\section{Author contributions}

PSP and KCB conceived the project and designed all experiments, with assistance from JLM for Western blot and CTZ experiments and CSM for the seizure behavioral model. PSP performed electrophysiology, calcium imaging, and histology experiments, as well as data analysis for all experiments. TJV and TOM conducted 2-photon calcium imaging and analyzed SD experiments. CSM conducted and analyzed seizure behavioral experiments. NOM and TOM performed SD experiments and analyzed baseline calcium activity. JLM conducted and analyzed Western blot experiments. PSP wrote the first draft of the manuscript. PSP and KCB are the primary authors of the manuscript, with assistance from TJV, CSM, NOM, and JLM. TJV and CSM share joint authorship.

\section{Acknowledgments}

We would like to thank our laboratory colleagues for valuable comments and suggestions. This work was supported by the United States Department of Defense (CDMRP PR 130373) and the NIH (R01NS085413).

Address correspondence to: Punam A. Sawant-Pokam and K.C. Brennan, 383 Colorow Drive Room 280A, Department of Neurology, Salt Lake City, Utah 84108, USA. Phone: 801.512.4282 (PSP), 801.581 .8129 (KCB); Email: punam.pokam@utah.edu, k.c.brennan@hsc.utah.edu. 
1. Langlois JA, Rutland-Brown W, Wald MM. The epidemiology and impact of traumatic brain injury: a brief overview. J Head Trauma Rehabil. 2006;21(5):375-378.

2. Xiong Y, Mahmood A, Chopp M. Animal models of traumatic brain injury. Nat Rev Neurosci. 2013;14(2):128-142.

3. Donkin J, Vink R. Mechanisms of cerebral edema in traumatic brain injury: therapeutic developments. Curr Opin Neurol. 2010;23(3):293-299.

4. Shah S, Kimberly WT. Today's approach to treating brain swelling in the neuro intensive care unit. Semin Neurol. 2016;36(6):502-507.

5. Cernak I, Wang Z, Jiang J, Bian X, Savic J. Ultrastructural and functional characteristics of blast injury-induced neurotrauma. J Trauma. 2001;50(4):695-706.

6. Talley Watts L, et al. Purinergic $2 \mathrm{Y} 1$ receptor stimulation decreases cerebral edema and reactive gliosis in a traumatic brain injury model. J Neurotrauma. 2013;30(1):55-66.

7. Dudek FE, Obenaus A, Tasker JG. Osmolalityinduced changes in extracellular volume alter epileptiform bursts independent of chemical synapses in the rat: importance of non-synaptic mechanisms in hippocampal epileptogenesis. Neurosci Lett. 1990;120(2):267-270.

8. Greer JE, Povlishock JT, Jacobs KM. Electrophysiological abnormalities in both axotomized and nonaxotomized pyramidal neurons following mild traumatic brain injury. J Neurosci. 2012;32(19):6682-6687.

9. Hanell A, Greer J, Jacobs K. Increased network excitability due to altered synaptic inputs to neocortical layer $\mathrm{V}$ intact and axotomized pyramidal neurons after mild traumatic brain injury. J Neurotrauma. 2015;32(20):1590-1598.

10. Carron SF, Alwis DS, Rajan R. Traumatic brain injury and neuronal functionality changes in sensory cortex. Front Syst Neurosci. 2016;10:47.

11. Lucke-Wold BP, et al. Traumatic brain injury and epilepsy: underlying mechanisms leading to seizure. Seizure. 2015;33:13-23.

12. Rao VR, Parko KL. Clinical approach to posttraumatic epilepsy. Semin Neurol. 2015;35(1):57-63.

13. Pietrobon D, Moskowitz MA. Chaos and commotion in the wake of cortical spreading depression and spreading depolarizations. Nat Rev Neurosci. 2014;15(6):379-393.

14. Fabricius M, et al. Cortical spreading depression and peri-infarct depolarization in acutely injured human cerebral cortex. Brain. 2006;129(3):778-790.

15. Strong A, et al. Spreading and synchronous depressions of cortical activity in acutely injured human brain. Stroke. 2002;33(12):2738-2743.

16. Dreier JP, et al. Spreading convulsions, spreading depolarization and epileptogenesis in human cerebral cortex. Brain. 2012;135(Pt 1):259-275.

17. Fabricius $\mathrm{M}$, et al. Association of seizures with cortical spreading depression and peri-infarct depolarisations in the acutely injured human brain. Clin Neurophysiol. 2008;119(9):1973-1984.

18. von Baumgarten L, Trabold R, Thal S, Back T, Plesnila N. Role of cortical spreading depressions for secondary brain damage after traumatic brain injury in mice. J Cereb Blood Flow Metab. 2008;28(7):1353-1360.
19. Van Harreveld A, Stamm JS. Spreading cortical convulsions and depressions. J Neurophysiol. 1953;16(4):352-366.

20. Koroleva V, Bures J. Cortical penicillin focus as a generator of repetitive spike-triggered waves of spreading depression in rats. Exp Brain Res. 1983;51(2):291-297.

21. Avoli M, et al. Epileptiform activity induced by low extracellular magnesium in the human cortex maintained in vitro. Ann Neurol. 1991;30(4):589-596.

22. Gorji A, Speckmann E-J. Spreading depression enhances the spontaneous epileptiform activity in human neocortical tissues. Eur J Neurosci. 2004;19(12):3371-3374.

23. Khoshkhoo S, Vogt D, Sohal VS. Dynamic, cell-type-specific roles for GABAergic interneurons in a mouse model of optogenetically inducible seizures. Neuron. 2017;93(2):291-298.

24. Bahari F, et al. Spreading depression and seizure unification experimentally observed in epilepsy [preprint]. Posted on bioRxiv October 29, 2018. https://doi.org/10.1101/455519.

25. Vespa PM, et al. Nonconvulsive seizures after traumatic brain injury are associated with hippocampal atrophy. Neurology. 2010;75(9):792-798.

26. Vespa PM, et al. Nonconvulsive electrographic seizures after traumatic brain injury result in a delayed, prolonged increase in intracranial pressure and metabolic crisis. Crit Care Med. 2007;35(12):2830-2836.

27. Hartings JA, et al. Spreading depolarizations have prolonged direct current shifts and are associated with poor outcome in brain trauma. Brain 2011;134(5):1529-1540.

28. Kharatishvili I, Nissinen JP, McIntosh TK, Pitkänen A. A model of posttraumatic epilepsy induced by lateral fluid-percussion brain injury in rats. Neuroscience. 2006;140(2):685-697.

29. Bareyre F, Wahl F, McIntosh T, Stutzmann J-M. Time course of cerebral edema after traumatic brain injury in rats: effects of riluzole and mannitol. J Neurotrauma. 1997;14(11):839-849.

30. Markgraf CG, Clifton GL, Moody MR. Treatment window for hypothermia in brain injury. J Neurosurg. 2001;95(6):979-983

31. Jha SK. Cerebral edema and its management. Med J Armed Forces India. 2003;59(4):326-331.

32. Chen T-W, et al. Ultrasensitive fluorescent proteins for imaging neuronal activity. Nature. 2013;499(7458):295-300.

33. Hall ED, et al. Spatial and temporal characteristics of neurodegeneration after controlled cortical impact in mice: more than a focal brain injury. J Neurotrauma. 2005;22(2):252-265.

34. Cantu D, et al. Traumatic brain injury increases cortical glutamate network activity by compromising GABAergic control. Cereb Cortex. 2015;25(8):2306-2320.

35. Vella J, Zammit C, Di Giovanni G, Muscat R, Valentino $\mathrm{M}$. The central role of aquaporins in the pathophysiology of ischemic stroke. Front Cell Neurosci. 2015;9:108.

36. Lafrenaye DA, Simard MJ. Bursting at the seams: molecular mechanisms mediating astrocyte swelling. Int J Mol Sci. 2019;20(2):330.

37. MacAulay N, Zeuthen T. Water transport between CNS compartments: contributions of aquaporins and cotransporters. Neuroscience. 2010;168(4):941-956.

38. Wang F, et al. NKCC1 up-regulation contributes to early post-traumatic seizures and increased post-traumatic seizure susceptibility. Brain Struct Funct. 2017;222(3):1543-1556.

39. Hui $\mathrm{H}$, et al. Inhibition of $\mathrm{Na}(+)-\mathrm{K}(+)-2 \mathrm{Cl}(-)$ cotransporter-1 attenuates traumatic brain injuryinduced neuronal apoptosis via regulation of Erk signaling. Neurochem Int . 2016;94:23-31.

40. Kahle KT, et al. Roles of the cation-chloride cotransporters in neurological disease. Nat Clin Pract Neurol. 2008;4(9):490-503.

41. Sooksawate T, Saito Y, Isa T. Electrophysiological and morphological properties of identified crossed tecto-reticular neurons in the rat superior colliculus. Neurosci Res. 2005;52(2):174-184.

42. Mongiat LA, Espósito MS, Lombardi G, Schinder AF. Reliable activation of immature neurons in the adult hippocampus. PLoS One. 2009;4(4):e5320.

43. Halabisky B, Parada I, Buckmaster PS, Prince DA. Excitatory input onto hilar somatostatin interneurons is increased in a chronic model of epilepsy. J Neurophysiol. 2010;104(4):2214-2223.

44. Neske GT. The slow oscillation in cortical and thalamic networks: mechanisms and functions. Front Neural Circuits. 2016;9:88.

45. Holcman D, Tsodyks M. The emergence of up and down states in cortical networks. PLOS Comput Biol. 2006;2(3):e23.

46. Sawant-Pokam P, Suryavanshi P, Dudek F, Brennan K. Mechanisms of neuronal silencing after cortical spreading depression. Cereb Cortex. 2017;27(2):1311-1325

47. Steriade M, Contreras D, Curró Dossi R, Nuñez A. The slow $(<1 \mathrm{~Hz})$ oscillation in reticular thalamic and thalamocortical neurons: scenario of sleep rhythm generation in interacting thalamic and neocortical networks. J Neurosci. 1993;13(8):3284-3299.

48. Petersen C, Crochet S. Synaptic computation and sensory processing in neocortical layer $2 / 3$. Neuron. $2013 ; 78(1): 28-48$.

49. Wang M, Ramos B, McCormick D. Alpha2A-adrenoceptors strengthen working memory networks by inhibiting cAMP-HCN channel signaling in prefrontal cortex. Cell. 2007;129(2):397-410.

50. Sanchez-Vives MV, McCormick DA. Cellular and network mechanisms of rhythmic recurrent activity in neocortex. Nat Neurosci. 2000;3(10):1027-1034.

51. Sanchez-Vives MV, et al. Inhibitory modulation of cortical up states. JNeurophysiol. 2010;104(3):1314-1324.

52. Constantinople CM, Bruno RM. Effects and mechanisms of wakefulness on local cortical networks. Neuron. 2011;69(6):1061-1068.

53. Neske GT, Patrick SL, Connors BW. Contributions of diverse excitatory and inhibitory neurons to recurrent network activity in cerebral cortex. JNeurosci. 2015;35(3):1089-1105.

54. Brandt C, Nozadze M, Heuchert N, Rattka M, Löscher W. Disease-modifying effects of phenobarbital and the NKCC1 inhibitor bumetanide in the pilocarpine model of temporal lobe epilepsy. J Neurosci. 2010;30(25):8602-8612.

55. Löscher W, Puskarjov M, Kaila K. Cation- 
chloride cotransporters NKCC1 and KCC2 as potential targets for novel antiepileptic and antiepileptogenic treatments. Neuropharmacology. 2013;69:62-74.

56. Lu K-T, et al. Inhibition of the $\mathrm{Na}^{+}-\mathrm{K}^{+}-2 \mathrm{Cl}^{-}-$ cotransporter in choroid plexus attenuates traumatic brain injury-induced brain edema and neuronal damage. Eur J Pharmacol. 2006;548(1):99-105.

57. Sivakumaran S, Maguire J. Bumetanide reduces seizure progression and the development of pharmacoresistant status epilepticus. Epilepsia. 2016;57(2):222-232.

58. Kaufmann D, Brennan KC. The effects of chronic stress on migraine relevant phenotypes in male mice. Front Cell Neurosci. 2018;12:294.

59. Otto JF, Yang Y, Frankel WN, Wilcox KS, White HS. Mice carrying the szt1 mutation exhibit increased seizure susceptibility and altered sensitivity to compounds acting at the m-channel. Epilepsia. 2004;45(9):1009-1016.

60. Otto JF, et al. Electroconvulsive seizure thresholds and kindling acquisition rates are altered in mouse models of human KCNQ2 and $\mathrm{KCNQ} 3$ mutations for benign familial neonatal convulsions. Epilepsia. 2009;50(7):1752-1759.

61. Rangel-Castilla L, Rangel-Castillo L, Gopinath S, Robertson CS. Management of intracranial hypertension. Neurol Clin. 2008;26(2):521-541.

62. Glykys J, Duquette E, Rahmati N, Duquette K, Staley KJ. Mannitol decreases neocortical epileptiform activity during early brain development via cotransport of chloride and water. Neurobiol Dis. 2019;125:163-175.

63. Pratt J, et al. The effect of riluzole and mannitol on cerebral oedema after cryogenic injury in the mouse. Neurosci Lett. 1999;272(3):143-146.

64. Kimelberg HK. Current concepts of brain edema. Review of laboratory investigations. J Neurosurg. 1995;83(6):1051-1059.

65. Sword J, Masuda T, Kirov SA, Croom D. Evolution of neuronal and astroglial disruption in the peri-contusional cortex of mice revealed by in vivo two-photon imaging. Brain .
2013;136(5):1446-1461.

66. Glykys J, et al. Chloride dysregulation, seizures, and cerebral edema: a relationship with therapeutic potential. Trends Neurosci. 2017;40(5):276-294.

67. Chamma I, Chevy Q, Poncer JC, Lévi S. Role of the neuronal $\mathrm{K}-\mathrm{Cl}$ co-transporter KCC2 in inhibitory and excitatory neurotransmission. Front Cell Neurosci. 2012;6:5.

68. $\mathrm{Wu} \mathrm{H}$, et al. Melatonin attenuates neuronal apoptosis through up-regulation of $\mathrm{K}(+)-\mathrm{Cl}(-)$ cotransporter KCC2 expression following traumatic brain injury in rats. J Pineal Res. 2016;61(2):241-250.

69. Wang F, et al. NKCC1 up-regulation contributes to early post-traumatic seizures and increased post-traumatic seizure susceptibility. Brain Struct Funct. 2017;222(3):1543-1556.

70. Lu K, Cheng N, Wu C, Yang Y. NKCC1-mediated traumatic brain injury-induced brain edema and neuron death via Raf/MEK/MAPK cascade. Crit Care Med. 2008;36(3):917-922.

71. Töpfer M, et al. Consequences of inhibition of bumetanide metabolism in rodents on brain penetration and effects of bumetanide in chronic models of epilepsy. Eur J Neurosci. 2014;39(4):673-687.

72. Kourdougli N, et al. Depolarizing $\gamma$-aminobutyric acid contributes to glutamatergic network rewiring in epilepsy. Ann Neurol. 2017;81(2):251-265.

73. Jayakumar AR, et al. Na-K-Cl cotransporter-1 in the mechanism of cell swelling in cultured astrocytes after fluid percussion injury. J Neurochem. 2011;117(3):437-448.

74. Hartings JA, et al. Spreading depolarisations and outcome after traumatic brain injury: a prospective observational study. Lancet Neurol. 2011;10(12):1058-1064.

75. Hartings JA, et al. Spreading depolarizations and late secondary insults after traumatic brain injury. J Neurotrauma. 2009;26(11):1857-1866.

76. Hartings JA. Spreading depolarization monitoring in neurocritical care of acute brain injury. Curr Opin Crit Care. 2017;23(2):94-102.
77. Glykys J, et al. Local impermeant anions establish the neuronal chloride concentration. Science. 2014;343(6171):670-675.

78. Bonislawski DP, Schwarzbach EP, Cohen AS. Brain injury impairs dentate gyrus inhibitory efficacy. Neurobiol Dis. 2007;25(1):163-169.

79. Chesnut RM, et al. A trial of intracranial-pressure monitoring in traumatic brain injury. $N$ Engl J Med. 2012;367(26):2471-2481.

80. Kilkenny C, Browne WJ, Cuthill IC, Emerson M, Altman DG. Improving bioscience research reporting: the ARRIVE guidelines for reporting animal research. PLoS Biol. 2010;8(6):e1000412.

81. Romine J, Gao X, Chen J. Controlled cortical impact model for traumatic brain injury. J Vis Exp. 2014;(90):e51781.

82. Kitamura K, Judkewitz B, Kano M, Denk W, Häusser M. Targeted patch-clamp recordings and single-cell electroporation of unlabeled neurons in vivo. Nat Methods. 2007;5(1):61-67.

83. Wilson C. Up and down states. Scholarpedia J. 2008;3(6):1410.

84. Pologruto TA, Sabatini BL, Svoboda K. ScanImage: flexible software for operating laser scanning microscopes. Biomed Eng Online. 2003;2(1):13.

85. Brennan KC, Romero-Reyes M, López Valdés HE, Arnold AP, Charles AC. Reduced threshold for cortical spreading depression in female mice. Ann Neurol. 2007;61(6):603-606.

86. Schneider CA, Rasband WS, Eliceiri KW. NIH Image to Image J: 25 years of image analysis. Nat Methods. 2012;9(7):671-675.

87. Theriot JJ, Toga AW, Prakash N, Ju YS, Brennan KC. Cortical sensory plasticity in a model of migraine with aura. J Neurosci. 2012;32(44):15252-15261.

88. Petersen CCH, Crochet S. Synaptic computation and sensory processing in neocortical layer $2 / 3$. Neuron. 2013;78(1):28-48.

89. Scholl EA, Dudek FE, Ekstrand JJ. Neuronal degeneration is observed in multiple regions outside the hippocampus after lithium pilocarpine-induced status epilepticus in the immature rat. Neuroscience. 2013;252:45-59. 